\title{
Radio emission from the massive stars in the galactic super star cluster Westerlund 1
}

\author{
S. M. Dougherty ${ }^{1,2}$, J. S. Clark ${ }^{3}$, I. Negueruela ${ }^{4}$, T. Johnson ${ }^{1,5}$, and J. M. Chapman ${ }^{6}$ \\ 1 National Research Council of Canada, Herzberg Institute for Astrophysics, Dominion Radio Astrophysical Observatory, \\ PO Box 248, Penticton, British Columbia V2A 6J9, Canada \\ e-mail: sean.dougherty@nrc.ca \\ 2 Institute for Space Imaging Science, University of Calgary, 2500 University Dr. NW., Calgary, Alberta, T2N 1N4, Canada \\ 3 Department of Physics and Astronomy, The Open University, Walton Hall, Milton Keynes, MK7 6AA, UK \\ 4 Dpto. de Física, Ingeniería de Sistemas y Teoría de la Señal, Universidad de Alicante, Apdo. 99, E03080 Alicante, Spain \\ 5 Department of Physics and Astronomy, University of Victoria, 3800 Finnerty Rd, Victoria, B.C., V8P 5C2, Canada \\ 6 Australia National Telescope Facility, PO Box 76, Epping, NSW 2121, Australia
}

Received 20 October 2009 / Accepted 16 November 2009

\section{ABSTRACT}

\begin{abstract}
Aims. Current mass-loss rate estimates imply that main sequence line-driven winds are not sufficient to strip away the H-rich envelope to yield Wolf-Rayet (WR) stars. The rich transitional population of the young massive cluster Westerlund 1 (Wd 1) provides an ideal laboratory to observe and constrain mass-loss processes throughout the transitional phase of stellar evolution.

Methods. We present an analysis of deep radio continuum observations of Wd 1 obtained with the Australia Telescope Compact Array at four frequency bands that permit investigation of the intrinsic characteristics of the radio emission.

Results. We detect 18 cluster members, a sample dominated by the cool hypergiants, with additional detections amongst the hotter OB supergiants and WR stars. The radio properties of the sample are diverse, with thermal, non-thermal and composite thermal/nonthermal sources present. Mass-loss rates determined for stars with partially optically thick stellar winds are $\sim 10^{-5} M_{\odot} \mathrm{yr}^{-1}$ across all spectral types, insufficient to enable the formation of WRs during a massive star lifetime, and the stars must undergo a period of greatly enhanced mass loss. The $\mathrm{sgB}[\mathrm{e}]$ star $\mathrm{W} 9$, the brightest radio source in $\mathrm{Wd} 1$, may provide an example, with a current mass-loss rate an order of magnitude higher than the other cluster members, and an extended nebula interpreted as a wind from an earlier epoch with a density $\sim 3 \times$ the current wind. Such an envelope structure in W9 is reminiscent of luminous blue variables, and one that shows evidence of two eras of high, possibly eruptive mass loss. Surprisingly, three of the OB supergiants are detected, implying unusually dense winds, though they are embedded in more extended emission regions that may influence the derived parameters. They also may have composite spectra, suggesting binarity, which can lead to a higher flux than expected from a stellar wind. Spatially resolved nebulae are associated with three of the four RSGs and three of the six YHGs in the cluster, which are due to quiescent mass loss rather than outbursts. The extended nebulae of W20 and W26 have a cometary morphology, implying significant interaction with either the intracluster medium or cluster wind. For some of the cool star winds, the ionizing source may be a companion star though the cluster radiation density is sufficiently high to provide the necessary ionizing radiation. Five WR stars are detected with composite spectra, interpreted as arising in colliding-wind binaries.
\end{abstract}

Key words. stars: evolution - H II regions - open clusters and associations: individual: Westerlund 1

\section{Introduction}

Recent work strongly suggests that canonical mass-loss rates for $\mathrm{O}$ stars need to be revised downwards to accommodate the effect of wind clumping (Fullerton et al. 2006; Mokiem et al. 2007). Such main sequence (MS) mass-loss rates are insufficient to remove the H-rich mantle of the star prior to it becoming a Wolf Rayet (WR), shifting the burden of mass loss onto the short lived transitional phase of stellar evolution. This phase is populated by a wide variety of highly luminous, hot supergiant $\mathrm{B}[\mathrm{e}]$ and luminous blue variable (LBV) stars, and cool Yellow Hypergiant (YHG) and red supergiant (RSG) stars. However the exact path of an $\mathrm{O}$ star through this transitional "zoo" as a function of initial mass is currently poorly understood, while the short-lived epochs, and hence rarity, of such stars complicates efforts to constrain their properties such as mass-loss rate and lifetime.

A better understanding of such short-lived phases in the life cycle of massive stars is crucial to areas of astrophysics other than just stellar evolution. For example massive stars are thought to predominantly form in stellar aggregates, where they drive cluster winds, which are a major source of mechanical feedback and chemically processed material into the wider galactic environment, in turn driving star formation and galactic evolution. Indeed the feedback from large populations of super star clusters (SSC; $M_{\text {total }} \sim 10^{5}-10^{7} M_{\odot}$ ) may drive galactic scale outflows, which, if present in Dwarf galaxies, may be sufficient to strip them of their interstellar medium, preventing subsequent generations of star formation (e.g. Westmoquette et al. 2007).

One such cluster in the Galaxy is Westerlund 1 (Westerlund 1961), hereafter Wd 1, for which photometric and spectroscopic observations suggest a unique population of both cool and hot supergiants (Borgman et al. 1970; Westerlund 1987). Recently, detailed optical and near-IR observations have confirmed these results and revealed $\mathrm{Wd} 1$ to be even more extreme than previously anticipated, containing a large population of post-MS stars with representative members of all evolutionary stages: OB supergiants and hypergiants, RSGs, YHGs and WRs (Clark \& Negueruela 2002; Clark et al. 2005, henceforth C02 and C05 respectively). Indeed, Wd 1 contains 6 YHGs, more than 50\% 
of the currently known population in the Galaxy, as well as one of the largest WR populations of any cluster in the Galaxy (Crowther et al. 2006). With a cluster mass of $\sim 10^{5} M_{\odot}(\mathrm{C} 05)$, Wd 1 is directly comparable to the SSCs observed in external galaxies such as M 82 and thus represents a relatively nearby example that provides a valuable opportunity to study the properties, evolution and interaction of massive stars in their "natural" environment.

Radio continuum observations are a long established tool for estimating mass-loss rates for early-type stars. As part of a programme to accomplish this for classical Be and B[e] stars, Clark et al. (1998, hereafter C98) imaged Wd 1 at radio wavelengths and found two unusually radio luminous stars; the $\operatorname{sgB}[\mathrm{e}]$ star W9 and the RSG W26. In both cases the emission was found to be spatially resolved, suggestive of recent mass-loss events. Motivated by these unusual radio properties and the possibility of detecting emission from massive post-MS stars over a broad range of evolutionary stages we carried out a more extensive radio observation of Wd 1 at four frequencies.

We present the results of this survey in this paper. The observations are described in Sect. 2, with the radio sources identified in Sect. 3. Section 4 discusses the nature of the radio sources, Sect. 5 is a brief discussion of the extended emission, and the results are summarized in Sect. 6.

\section{Observations}

Observation of Wd 1 have been obtained at 8640, 4800, 2496 and $1384 \mathrm{MHz}$ (corresponding to 3,6, 13 and $20 \mathrm{~cm}$ respectively) using the Australia Telescope Compact Array. A first epoch was obtained 1998 March 4-5 in the 6B configuration, followed by observations 2001 January 7-8 in the more compact $750 \mathrm{C}$ configuration and on 2002 May 18 (only 8640 and $4800 \mathrm{MHz}$ ) in the $6 \mathrm{~A}$ configuration. Together, these observations ensure good coverage across the spatial frequency range observed, though do not have identical spatial frequency coverage at each frequency. Hence, caution is suggested when comparing flux measures at the different frequencies for resolved, extended emission.

Observations of Wd 1 were interleaved with repeated observations of the nearby bright point source 1657-56 in order to establish the antenna-based complex gains. Initial editing and calibration of all observations was done using the MIRIAD data reduction package (Sault et al. 1995). The gain solutions established using 1657-56 were subsequently "referenced" by interpolation to the Wd 1 observations. The absolute flux scale was established using the primary calibrator 1934-638, assuming fluxes of $2.84,5.83,11.14$ and $14.94 \mathrm{Jy}$ at $8.6,4.8,2.5$ and $1.4 \mathrm{GHz}$ respectively. The fluxes derived for $1657-56$ are shown in Table 1. No significant flux variations $(>10 \%$ of the source flux) were noted in preliminary analysis of the fluxes of the brightest unresolved sources $(>2 \mathrm{mJy}$ at $8.6 \mathrm{GHz}$ ) in data from each epoch. Hence, data from the three epochs were combined into one dataset in order to improve the signal-to-noise ratio of the data and improve our ability to detect weaker sources.

After phase-referencing and subsequent combination of the data from each epoch, deconvolution was done through visibility model fitting, a technique widely used in VLBI image construction, rather than the commonly used CLEAN technique. This was done using an automated model-fitting routine, the MODCONS macro within the SMERF patch (Reid 2006) to the DIFMAP package (Shepherd 1997). The major advantage of the "smear fitting" method implemented by SMERF is that it yields higher resolution for significantly detected features than estimated by the
Table 1. Fluxes determined for the phase-reference 1657-56.

\begin{tabular}{llllll}
\hline \hline & Config. & $\begin{array}{l}8.6 \mathrm{GHz} \\
(\mathrm{Jy})\end{array}$ & $\begin{array}{l}4.8 \mathrm{GHz} \\
(\mathrm{Jy})\end{array}$ & $\begin{array}{l}2.5 \mathrm{GHz} \\
(\mathrm{Jy})\end{array}$ & $\begin{array}{l}1.4 \mathrm{GHz} \\
(\mathrm{Jy})\end{array}$ \\
\hline 1998 Mar. & 6B & 1.54 & 1.81 & 1.92 & 1.89 \\
2001 Jun. & $750 \mathrm{C}$ & 1.44 & 1.61 & 1.71 & 1.88 \\
2002 May & $6 \mathrm{~A}$ & 1.63 & 1.79 & & \\
\hline
\end{tabular}

oft-used Rayleigh criteria (Reid 2006), represented by the FWHM of the synthesized beam. A model was established for the 8.6-GHz visibilities consisting of point and Gaussian source models, using the image data to guide the modelling process. This gave a model at the highest observed frequency, and hence at the highest resolution. Once the positions of the model components had been determined, the relatively high signal-to-noise ratio of the brightest sources in the field permitted the use of phaseonly self-calibration to improve the antenna gain solutions from those derived from phase referencing alone. A "best" model was established with a final round of model fitting, keeping the positions fixed, to refine the flux of the model components. Note that no amplitude self-calibration was carried out using these models. This "best" model at $8.6 \mathrm{GHz}$ was then used as the input model at the other frequencies. The resulting images at the four passbands are shown in Fig. 1.

\section{The radio sources in $\mathrm{Wd} 1$}

\subsection{Identification of radio stars}

In order to identify stellar counterparts to the radio sources in Wd 1, an $R$-band image (655 nm effective wavelength) of the cluster was obtained on 2004 June 13 using the FORS2 camera on the VLT. After re-projecting the optical image to the same projection as the ATCA images using the AIPS task OHGEO, the absolute position of the optical image was fixed by assuming that the position of the point source component of the 8.6-GHz emission from W9, the brightest source in the radio image, is coincident with the peak of its optical emission, as determined using AIPS routine MAXFIT. Since the position of 8.6-GHz emission components was established by phase-referencing, the absolute position accuracy of the model components is determined by the accuracy of the position of the phase-reference source 1657-56, and on the residual antenna gain phase as a result of the offset between the position of the phase-reference source and $\mathrm{Wd} 1$, separated by 8.8 degrees. It is estimated this leads to an uncertainty of $\sim 100$ mas in the absolute positions of the radio sources (see Table 2).

The resulting overlay of the 8.6-GHz image and the $R$-band image is shown in Fig. 2. A large number of optical sources are coincident with radio sources. Setting a $4 \sigma$ point source detection limit of $0.23,0.43,0.86$ and $1.45 \mathrm{mJy} \mathrm{beam}^{-1}$ at 8.6, 4.8, 2.5 and $1.4 \mathrm{GHz}$ respectively, we identify the optical sources with associated radio emission in Table 2.

The position of putative optical counterparts to the radio sources were also determined using the AIPS routine MAXFIT, and an offset between the radio and optical positions derived. The uncertainty in the relative position of the radio and the optical image is governed by how well the optical image is referenced to the radio image. By inspection of the position of W9, we estimate this to be an uncertainty of approximately \pm 200 mas. This is the dominant position uncertainty for the sources. Radio sources that have a position offset from a putative optical "counterpart" greater than 600 mas may not be 


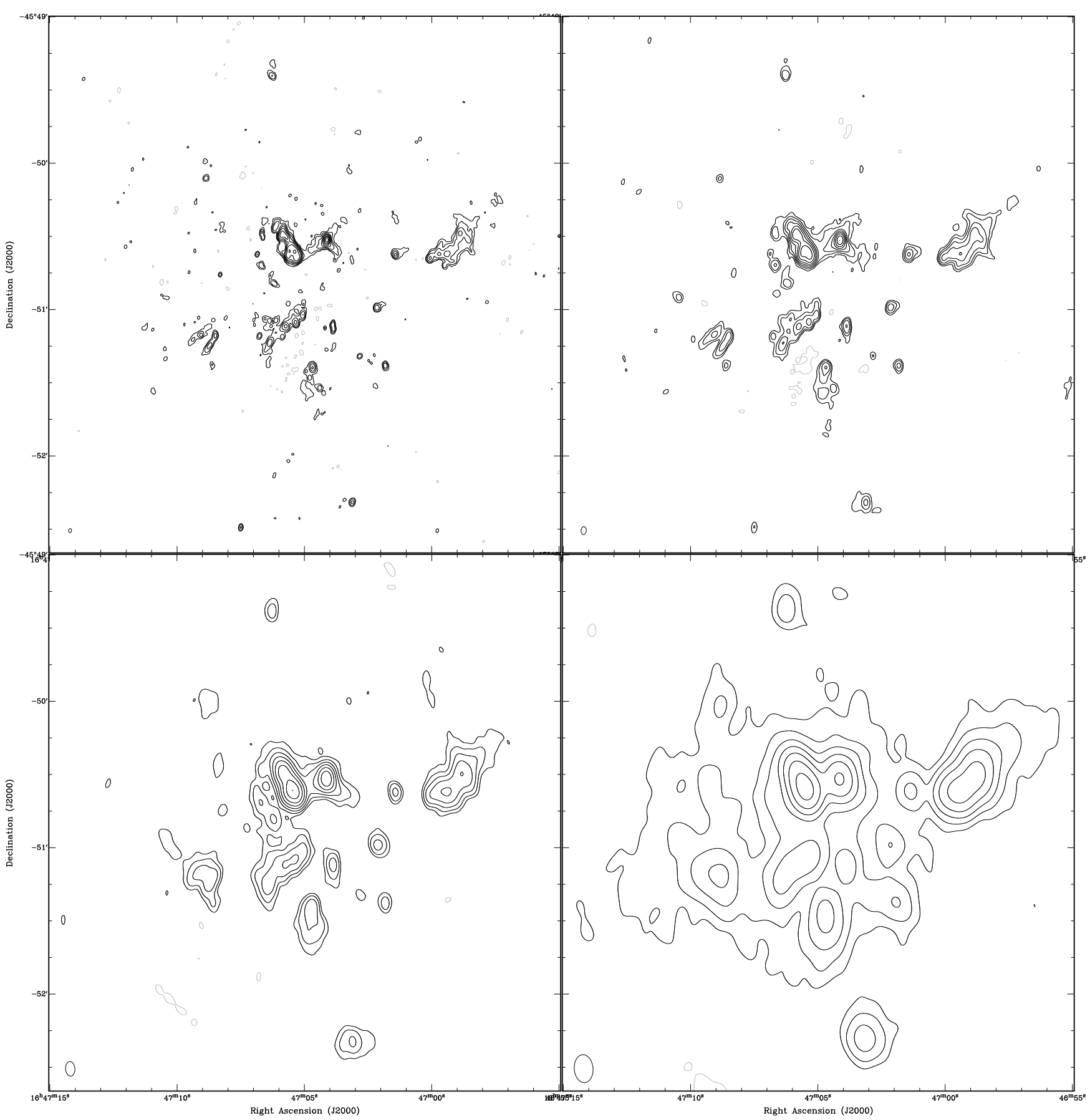

Fig. 1. The ATCA observations of Wd 1 at $8.6 \mathrm{GHz}$ (upper left), $4.8 \mathrm{GHz}$ (upper right), $2.5 \mathrm{GHz}$ (lower left) and $1.4 \mathrm{GHz}$ (lower right). In each image, contour levels are $-3,3,6,12,24,48,96,192 \sigma$, where the $1 \sigma \mathrm{rms}$ uncertainty is $0.06,0.1,0.2,0.4 \mathrm{mJy}_{\text {beam }}{ }^{-1}$ respectively at the four frequencies. These images were reconstructed using the nominal FWHM of the synthesized beam at each frequency, shown in the lower left-hand corner of each image. Note that these images are not corrected for the primary beam pattern.

associated, and so these potential mis-identifications are identified in Table 2.

A total of 18 stars are identified as radio emitters, with optical-radio positional offsets $<0.6^{\prime \prime}$. We identify two objects with associated or potentially associated radio emission for which no designation is available in the literature. Spectroscopy that could identify if they are bona-fide cluster members is not available for these objects so we do not assign Westerlund numbers to them, but name them as D09-R1 and D09-R2 following the convention used by Clark et al. (2008, hereafter C08). Three stars are identified as possible radio emitters (W5, W16a and D09-R2) since they are offset from the potential radio "counterpart" by between 0.6 and 1.0 aresed, and somo caution may be warranted identifying these stars with the nearby radio emission.

With this many stellar radio sources, Wd 1 has the richest population of radio emitting stars known for any young massive galactic cluster (e.g. Lang 2003; Moffat et al.2002; Setia Gunawan et al. 2003). The stellar radio sources are blue, 


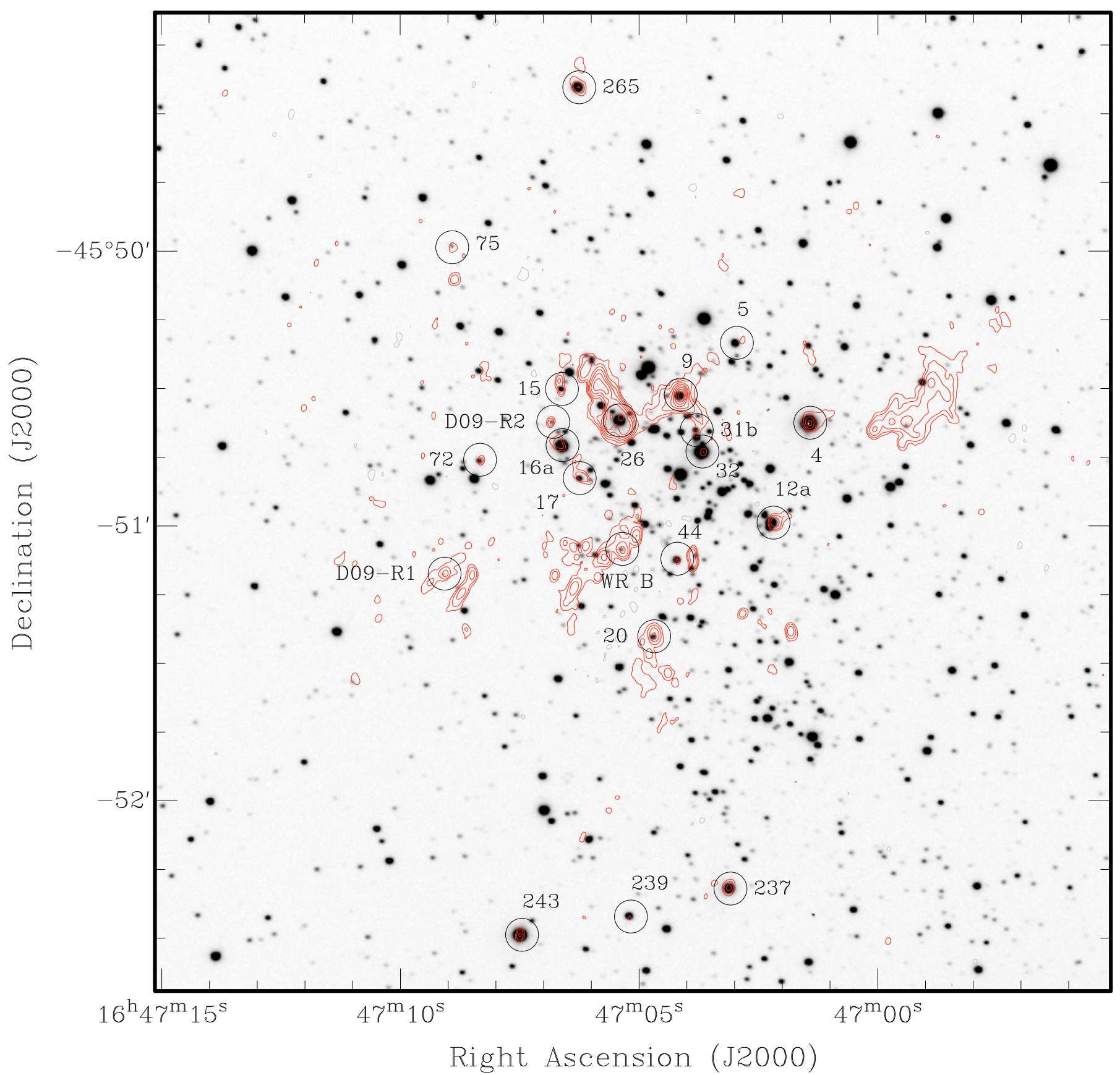

Fig. 2. 8.6-GHz image overlaid on a FORS $R$-band image. The limiting magnitude of the $R$-band image is $\approx 17.5$ mag. The radio data are represented by contours with levels at $-3,3,6,12,24,48,96,192 \sigma$, where the $1 \sigma \mathrm{rms}$ uncertainty is $0.06 \mathrm{mJy}_{\text {beam }}^{-1}$. The radio sources with putative optical counterparts listed in Table 2 are identified by the circles and Westerlund numbers.

yellow or red super- or hypergiants and WR stars, representative of different stages of massive star evolution. The supergiant B[e] star W9 is by far the brightest stellar radio emitter in the cluster, as anticipated from the "snapshot" observation of the cluster presented by C98. Among the other four known blue hypergiants, only the LBV W243 is detected. Wd 1 has one of the richest populations of YHGs known in the Milky Way, with six members of this group (W4, 8a, 12a, 16a, 32, and 265), all of which are detected except for W8a and possibly W16a. All four known RSGs, six of the 16 WR stars in the field-of-view (24 known currently in the cluster), and four OB supergiants, are also associated with radio emission.

A number of sources appear to consist of multiple components (W4, W9, D09-R1 and W237) with a central unresolved source surrounded by a more extended component that is often significantly larger than the synthesized beam of the array. For W4 and W9, the extended component is clearly centred on the stellar source and an association with the extended emission is strongly implied. For D09-R1, the relationship of the stellar source and the extended emission is not as clear since the extended emission is offset from the associated point source.

\subsection{Radio star characteristics}

The radio fluxes of the sources were deduced from the model components derived in the model fitting process, which were then corrected for attenuation by the primary beam of the ATCA, dependent on their distance from the pointing centre. 
S. M. Dougherty et al.: Radio emission from the massive stars in the galactic super star cluster Westerlund 1

Table 2. Characteristics of stellar radio sources in the $\mathrm{Wd} 1$ cluster.

\begin{tabular}{|c|c|c|c|c|c|c|c|c|c|c|}
\hline $\begin{array}{l}\text { Source ID } \\
a, b\end{array}$ & $\begin{array}{c}\text { Spectral } \\
\text { Type }^{c}\end{array}$ & 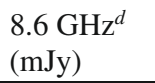 & $\begin{array}{l}\begin{array}{l}4.8 \mathrm{GHz} \\
(\mathrm{mJy})\end{array} \\
\end{array}$ & $\begin{array}{l}\begin{array}{l}2.5 \mathrm{GHz} \\
(\mathrm{mJy})\end{array} \\
\end{array}$ & $\begin{array}{l}1.4 \mathrm{GHz} \\
(\mathrm{mJy})\end{array}$ & Spec. Index & $\begin{array}{l}\text { Radio } \\
16^{\mathrm{h}} 47^{\mathrm{m}}\end{array}$ & $\begin{array}{l}\text { pos. }^{e} \\
-45^{\circ}\end{array}$ & $\begin{array}{l}\Delta^{f} \\
\left({ }^{\prime \prime}\right)\end{array}$ & Notes \\
\hline $4(\mathrm{H})$ & $\mathrm{F} 2 \mathrm{Ia}^{+}$ & $0.8 \pm 0.08$ & $0.6 \pm 0.1$ & $33+03^{9}$ & $38+04$ & $+0.49 \pm 0.31$ & 1.445 & 5037.66 & 0.37 & \\
\hline 4 & F2 1a & $2.2 \pm 0.2 \mathrm{r}^{h}$ & $2.8 \pm 0.3 r$ & $3 .$. & 3. & $-0.23 \pm 0.07$ & & & & 1 \\
\hline $9(\mathrm{C})$ & $\mathrm{sgB}[\mathrm{c}$ & $24.9 \pm 2.5$ & $18.1 \pm 1.8$ & $9.5 \pm 0.9$ & $7.8 \pm 0.8$ & $+0.68 \pm 0.07$ & 4.136 & 5031.41 & & \\
\hline 9 & & $30.5 \pm 3.0 \mathrm{r}$ & $28.4 \pm 2.8 \mathrm{r}$ & $21.0 \pm 2.1 \mathrm{r}$ & $24.5 \pm 2.5 \mathrm{r}$ & $+0.16 \pm 0.07$ & & & & \\
\hline $12 \mathrm{a}$ & A $5 \mathrm{Ia}^{+}$ & $2.9 \pm 0.3 r$ & $2.9 \pm 0.3$ & $3.5 \pm 0.4$ & $3.0 \pm 0.4$ & $-0.06 \pm 0.08$ & 2.151 & 5059.23 & 0.61 & 1 \\
\hline 15 & O9.5-B0.5Ia & $0.6 \pm 0.06$ & $0.7 \pm 0.1$ & $<0.6^{i}$ & $<1.1$ & $>0.0$ & 6.648 & 5030.31 & 0.54 & \\
\hline 17 & O9.5-B0.5Ia & $1.7 \pm 0.2$ & $1.6 \pm 0.2$ & $3.2 \pm 0.3$ & $2.9 \pm 0.4$ & $-0.42 \pm$ & 6.183 & 5049.40 & 0.50 & \\
\hline WR B & WN7 & $4.3 \pm$ & $4.8=$ & 4. & $4 .(1$ & +0.04 & 5.345 & $51 \quad 5.21$ & 0.21 & 2 \\
\hline 20 & RSG & $3.8=$ & $4.2=$ & .4 & 4.8 & $-0.11=$ & 4.679 & 5123.81 & 0.33 & 2 \\
\hline $26(\mathrm{~A})$ & $\mathrm{R}$ & 20.1 & 21.9 & $18.4 \pm 1.8 \mathrm{r}$ & ? & $+0 .($ & 5.370 & 5036.57 & 0.33 & 2 \\
\hline $31 \mathrm{~b}(\mathrm{WR} V)$ & WN8 & $0.4 \pm$ & $0.4 \pm 0.1$ & $<0.6$ & $<1$. & $0.00=$ & 3.821 & 5039.22 & 0.45 & \\
\hline 32 & $\mathrm{~F} 5 \mathrm{Ia}^{+}$ & $0.4 \pm$ & $0.4 \pm 0.1$ & $<0.6$ & $<1.1$ & $0.00 \pm 0.50$ & 3.675 & 5043.70 & 0.31 & \\
\hline 44(WR L) & WN9 & $0.4 \pm 0.06$ & $<0.3$ & $<0.6$ & $<1.1$ & $>0.5$ & 4.210 & 517.34 & 0.23 & \\
\hline D09-R1 & & $0.7 \pm 0.07$ & $0.8 \pm 0.1$ & & & $-0.23 \pm 0.27$ & 9.075 & 5110.23 & 0.31 & \\
\hline D09-R1? & B & $6.5 \pm 1.2 \mathrm{r}$ & $6.2 \pm 1.2 \mathrm{r}$ & & 1 & $-0.61 \pm 0.11$ & & & & 1,3 \\
\hline 72(WR A) & WN7 & $0.5 \pm 0.06$ & $0.5 \pm 0.1$ & $<0.6$ & $<1.1$ & $0.00 \pm 0.40$ & 8.332 & 5045.58 & 0.00 & \\
\hline 75 & RSG & $0.3 \pm 0.06$ & $<0.3$ & $<0.6$ & $<1.1$ & $>0.0$ & 8.933 & 4958.92 & 0.46 & \\
\hline 237(B) & RSG & $1.8 \pm 0.2$ & $1.8 \pm 0.2$ & $1.9 \pm 0.2$ & $2.0 \pm 0.4$ & $-0.06 \pm 0.10$ & 3.117 & 5218.99 & 0.28 & \\
\hline 237 & & $5.6 \pm 2.2 \mathrm{r}$ & $7.5 \pm 2.1 \mathrm{r}$ & $10.6 \pm 3.0 \mathrm{r}$ & $11.2 \pm 1.9 \mathrm{r}$ & $-0.35 \pm 0.19$ & & & & 1,3 \\
\hline 239(WR F) & WC9d & $0.3 \pm 0.06$ & $<0.3$ & $<0.6$ & $<1.1$ & $>0.0$ & 5.196 & 5225.63 & 0.50 & \\
\hline 243(G) & LBV & $1.5 \pm 0.2$ & $0.9 \pm 0.1$ & $<0.6$ & $<1.1$ & +0.87 & 7.496 & 5229.28 & 0.32 & \\
\hline $265(\mathrm{I})$ & F5 $\mathrm{Ia}^{+}$ & $2.3 \pm 0.3 r$ & $2.1 \pm 0.2$ & $1.7 \pm 0.2$ & $1.8 \pm 0.4$ & $+0.20 \pm 0.11$ & 6.268 & 4924.19 & 0.30 & \\
\hline 5(WR S) & WN10-11 & $0.3 \pm 0.06$ & $<0.3$ & $<0.6$ & $<1.1$ & $>0.0$ & 2.877 & 5019.76 & 0.91 & 4 \\
\hline $16 a(L)$ & A2 $\mathrm{Ia}^{+}$ & $1.6 \pm$ & $2.0 \pm 0.2$ & $2.8 \pm$ & $3.0 \pm$ & $-0.33 \pm 0.09$ & 6.688 & 5041.99 & 0.93 & 4 \\
\hline D09-R2 & BSG & $0.7 \pm 0.06$ & $0.8 \pm 0.1$ & $1.2 \pm 0.2$ & $1.6 \pm 0.4$ & $-0.43 \pm 0.11$ & 6.867 & 5037.17 & 0.70 & 4 \\
\hline
\end{tabular}

Notes. 1) Extended component. 2) These sources are embedded in extended emission which may influence the flux estimates. 3) Not clear if the extended emission is related to the compact radio source or the underlying optical source. 4) The radio emission is offset from the stellar position by more than $3 \times$ the position uncertainty introduced by phase-transfer i.e. 0.6 arcsec. Hence, association of radio emission with the optical source is uncertain.

(a) Source numbers from Westerlund (1987). ${ }^{(b)}$ Alternate names from C02, Borgman et al. (1970), Negueruela \& Clark (2005), and Crowther et al. (2006). ${ }^{(c)}$ Spectral types from C02, C05, Negueruela \& Clark (2005) and Crowther et al. (2006). ${ }^{(d)}$ Fluxes derived from visibility modelling, corrected for the primary beam of the ATCA. ${ }^{(e)} \mathrm{J} 2000$ positions of model components at $8.6 \mathrm{GHz}$, assuming reference source $1657-56$ at $\alpha=17: 01: 44.853, \delta=-56: 21: 55.964$. The uncertainty in absolute radio position is estimated to be \pm 0.1 arcsec. ${ }^{(f)}$ Separation of the optical and radio positions. ${ }^{(g)}$ For sources with two components at higher frequencies, when there is only one model component determined this represents a total flux at this frequency. ${ }^{(h)}$ The symbol " $\mathrm{r}$ " denotes components that appear resolved by the ATCA at that frequency. ${ }^{(i)}$ Quoted upper limits are $3 \sigma$.

The FWHM of the ATCA primary beam is 5.86, 10.06, 19.91 and 34.61 arcmin at 8.6, 4.8, 2.5 and $1.4 \mathrm{GHz}$ respectively. Given the size of the field-of-view is $\sim 4$ arcmin, this leads to flux corrections of up to $1.4 \times$ in the outer regions of the field-of-view at $8.6 \mathrm{GHz}$. In an attempt to allow for systematic errors, the flux uncertainties quoted in Table 2 are taken from the maximum of the following three values: the $1 \sigma \mathrm{rms}$ uncertainty in the image, $10 \%$ of the source flux, or for resolved sources the rms uncertainty in the image multiplied by the solid angle of the source. This represents a conservative approach to estimating the flux uncertainties, and hence to the estimated spectral indices. However, it presumes that the model components used to describe a source are not influenced by other emission from the region surrounding the source. This may be an additional complication in determining the fluxes of several sources (WR B, W20 and W26) since they are embedded in more extended regions of emission that may impact the accuracy of the derived fluxes. In addition to the source flux, it is noted which sources appear resolved from the visibility modelling directly, avoiding the impact of visibility weighting during imaging on the CLEAN beam size and the subsequent limitations of source modelling in the image plane.

It is noted that the flux values determined in this study are approximately $10 \%$ higher than the values previously reported by C98. It is not clear if the apparent increase in the flux of W9 is due to intrinsic source variations or due to the absolute calibration scale, given that the uncertainty in the absolute flux calibration is $\sim 5-10 \%$. We suspect the apparent variation is due to a combination of absolute calibration uncertainty and the fact the data used in this study extend to lower spatial frequencies than in the study of C98, and hence may recover flux that was "resolved out" in the earlier observations. We suggest a more accurate determination of total flux is presented here than in the previous work.

The radio spectral index $\alpha$, where $S_{v} \propto v^{\alpha}$, for each source was calculated by a weighted regression fit of a single powerlaw to the fluxes. Use of a single power-law presumes there is no curvature in the continuum spectra over the frequency range observed. Inspection of the spectra of those sources detected at all bands shows no compelling evidence of curvature, given the uncertainties in the radiometry at each band. The resulting values for spectral indices and uncertainties are given in Table 2 and displayed in Fig. 3. For sources with radiometry at all frequency bands, the resulting single power-law index values are less affected by potential systematic errors in the flux measures at each band, compared to indices derived across a smaller frequency range e.g. 8.6 to $4.8 \mathrm{GHz}$. For sources that are undetected at one or more bands, and upper limits quoted, only the detected fluxes were used to calculate spectral index, unless they helped further 
Table 3. Number of radio emitters of given spectral type.

\begin{tabular}{lccc}
\hline \hline $\begin{array}{l}\text { Spec. } \\
\text { Type }\end{array}$ & $\begin{array}{c}\text { No. radio } \\
\text { emitters }\end{array}$ & $\begin{array}{c}\text { Source } \\
\text { ID }\end{array}$ & $\begin{array}{c}\text { Cluster } \\
\text { total }\end{array}$ \\
\hline OB SGs & $3(4 ?)^{a}$ & 15,17, D09-R1, (D09-R2?) & $\sim 150$ \\
sgB[e] & 1 & 9 & 1 \\
BHGs & 1 & 243 & 4 \\
YHGs & $4(5 ?)^{a}$ & $4,12 \mathrm{a}, 32,265(16 \mathrm{a} ?)$ & 6 \\
RSGs & 4 & $20,26,75,237$ & 4 \\
WN9-10 & $1\left(2 ?^{a}\right.$ & $44,(5)$ & 2 \\
WN5-8 & 3 & WR B, 31b, 72 & 14 \\
WC & 1 & 239 & 8 \\
\hline
\end{tabular}

Notes. ${ }^{(a)}$ These sources are included in parentheses due to the uncertain association of radio emission with W16a, D09-R2 and W5. ${ }^{(b)}$ WRs N (WC8), O and Q (both WNL) are not in the field-of-view of the radio observations described here.

constrain the spectral index value e.g. W44. It should be noted that the fluxes for sources only detected at one or two bands are all low $(<1 \mathrm{mJy})$ and the uncertainty in the derived spectral indices is high.

None of the sources have a completely optically-thick thermal spectrum ( $\alpha \sim 2)$, though several have spectra consistent with $\alpha \sim+0.6$, expected for a partially optically-thick, steadystate stellar wind e.g. the compact components in W4, 9, 44 and 243 .

The majority of the sources have indices that are quite shallow compared to the expected value for a stellar wind, with many having indices consistent with -0.1 , the value for opticallythin thermal emission. A combination of optically thick and thin emission components can give rise to such spectra, such as clumpy stellar winds where the clumps are optically thick (e.g. Ignace \& Churchwell 2004). Alternatively, in some of these objects the emission may be due to a combination of thermal and non-thermal emission forming a composite spectrum, as observed in a number of massive stars, where the non-thermal emission is attributed to a colliding-wind binary (CWB) (e.g. Chapman et al. 1999; Dougherty \& Williams 2000; van Loo et al. 2006). Depending on the relative strength of the two continuum components, the spectral index could lie anywhere between $\sim+0.8$ and -0.6 , this latter value typical of optically-thin synchrotron emission. This makes an unequivocal identification of the underlying emission difficult. To compound matters, composite spectra due to thermal+non-thermal emission may not be well represented by a single power-law (e.g. Pittard et al. 2006). However, identification of CWBs is helped through observations at other wavelengths, largely thermal X-ray emission arising from shock-heated plasma in the wind-collision region, and/or dust emission that appears to be a common feature in carbon-type WR star massive binaries with non-thermal emission (Williams 1999).

There are a few sources with indices that, at face value, are more negative than -0.1 , suggesting non-thermal emission e.g. W17 and the extended component associated with W4. Other potential members of this group are W16a, D09-R2 and the extended emission near D09-R1 but, as noted earlier, the association of the radio emission with the underlying stars is unclear. The derived spectral index for the extended emission in W4 is consistent with optically- thin thermal emission at the $95 \%$ confidence level. This leaves W17 as the only bona-fida non-thermal source.

Additional observations over a broader range of frequencies are required to establish more firmly the nature of the radio

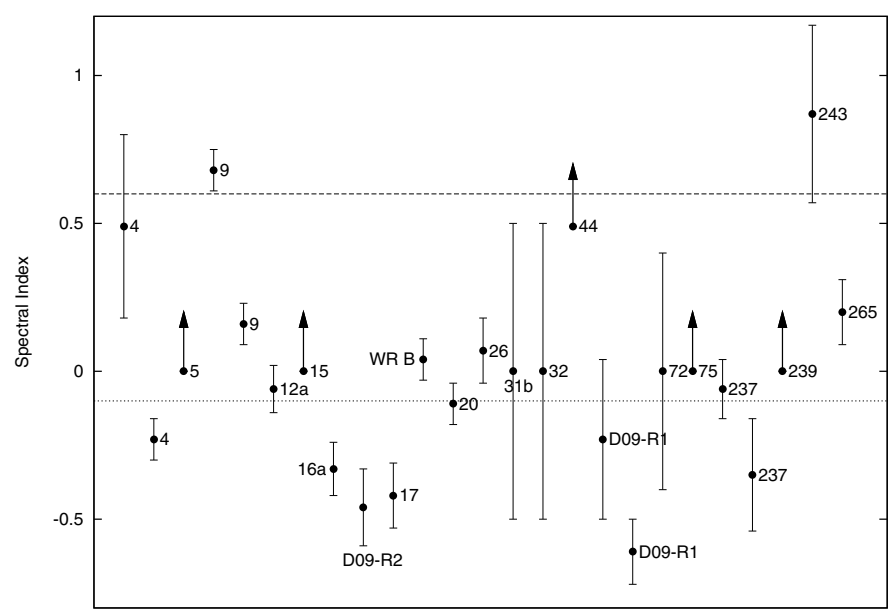

Fig. 3. The spectral indices of the stellar radio sources in Wd 1, identified by their Westerlund numbers. The canonical spectral indices for partially optically thick thermal emission from a stellar wind $(+0.6)$ and optically thin thermal emission $(-0.1)$ are highlighted by the dashed and dotted lines. Error bars are 1 sigma. The arrows denote lower limits to the derived spectral index. Radio sources offset from potential optical counterparts by more than $0.6^{\prime \prime}$ are marked by open boxes.

Table 4. Properties of the extended radio emission regions in the cluster centre.

\begin{tabular}{llllll}
\hline \hline ID & $\begin{array}{l}8.6 \mathrm{GHz} \\
(\mathrm{mJy})\end{array}$ & $\begin{array}{l}4.8 \mathrm{GHz} \\
(\mathrm{mJy})\end{array}$ & $\begin{array}{l}2.5 \mathrm{GHz} \\
(\mathrm{mJy})\end{array}$ & Spec. Index & $\begin{array}{l}\text { Assoc. } \\
\text { Star }\end{array}$ \\
\hline A1 & 5.6 & 7.6 & 7.6 & $-0.24 \pm 0.15$ & D09-R1 \\
A2 & 23.8 & 26.0 & 35.5 & $-0.32 \pm 0.10$ & WR B \\
$\mathrm{A} 3$ & 12.1 & 11.1 & 16.0 & $-0.23 \pm 0.20$ & W20 \\
A4 & 3.5 & 4.7 & 5.5 & $-0.36 \pm 0.10$ & \\
A5 & 2.0 & 1.9 & 2.0 & $-0.01 \pm 0.11$ & \\
A6 & 71.9 & 78.0 & 81.8 & $-0.05 \pm 0.07$ & \\
A7 & 22.4 & 28.2 & 27.0 & $-0.14 \pm 0.13$ & W9 \\
A8 & 153.3 & 164.9 & 161.4 & $-0.04 \pm 0.11$ & W26 \\
Total & 295 & 322 & 337 & & \\
\hline
\end{tabular}

Notes. These fluxes are for the extended emission only in these regions. The fluxes of the radio stars in these regions has been subtracted. The flux uncertainty is assumed to be $\sim 10 \%$.

emission from the stars in $\mathrm{Wd} 1$. Individual cases are discussed in Sect. 4 where the observations from each stellar sub-type are discussed in detail.

\subsection{Extended radio emission}

In addition to the radio emission that is associated with stars it is clear there are a number of large, extended emission regions, distributed within $\sim 1.5 \mathrm{arcmin}$ of the cluster centre. Some of these regions appear to be associated with stars e.g. A3 with W20 and A8 with W26, but there are others such as A5 and A6 for which optical counterparts are not identified in the FORS2 image. These regions are identified in Fig. 4 and the radio properties summarised in Table 4. The spatial frequency coverage is not the same at all frequencies and some extended emission may be resolved out at $8.6 \mathrm{GHz}$ and hence the reported fluxes may be low relative to the fluxes at lower frequencies. Hence, some of the spectral indices reported for the individual regions in Table 4 may actually be flatter, or less negative, than reported.

The total radio flux from $\mathrm{Wd} 1$ as measured in the interferometer data presented here is 422, 461, 523 and $669 \mathrm{mJy}$ 


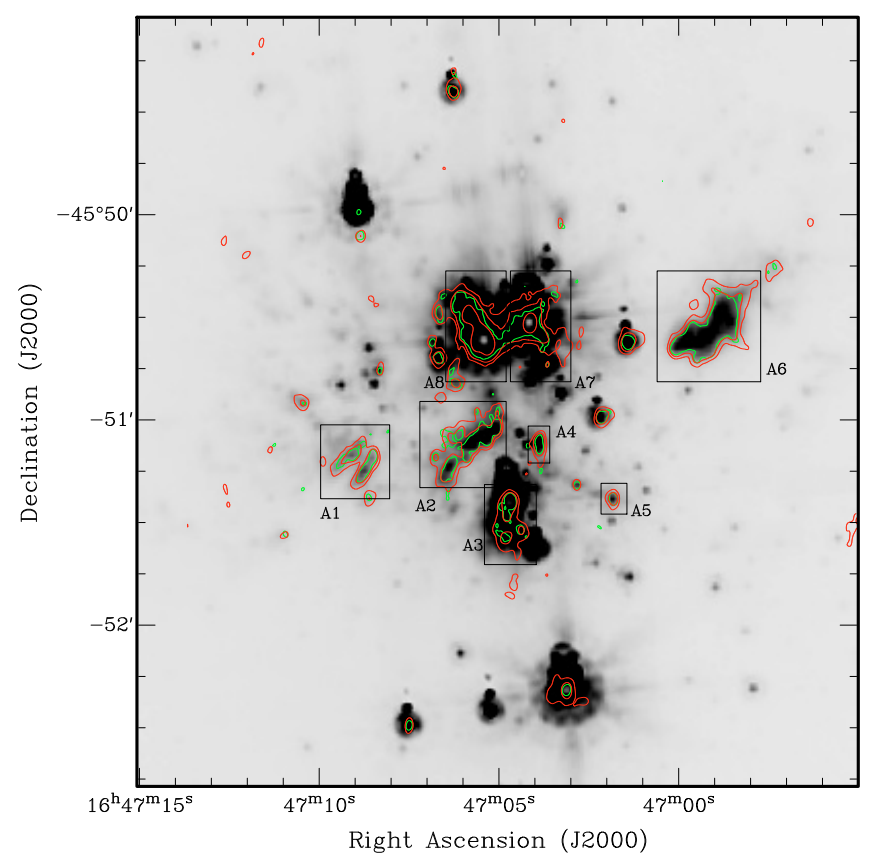

Fig. 4. Comparison of radio emission at 8.6 (green) and 4.8-GHz (red) observations with $8 \mu \mathrm{m}$ emission taken as part of the Spitzer GLIMPSE project. Only a few radio contours that demark the extended radio emitting regions are shown for clarity.

at $8.6,4.8,2.2$, and $1.4 \mathrm{GHz}$ respectively. This is compared to an interpolation of the single-dish fluxes reported by Kothes \& Dougherty (2007), which gives fluxes at the same frequencies of $450,499,560$, and $620 \mathrm{mJy}$. Given that the flux uncertainties in the single dish measures is likely $\sim 10 \%$, the total fluxes derived here from the ATCA data are in excellent agreement with the single dish fluxes.

To determine the flux in extended emission, we subtract the radio emission associated with the stellar sources as given in Table 2 from the total flux determined by the ATCA. This leads to fluxes of 307,351 and $426 \mathrm{mJy}$ at $8.6,4.8$ and $2.2 \mathrm{GHz}$, with an uncertainty of $\sim 5-10 \%$. The flux at $1.4 \mathrm{GHz}$ is not quoted since it is difficult to ascertain accurately for the radio stars, most especially for sources embedded in extended emission. Comparing these values to those given in Table 4 and taking into account their uncertainty, shows good agreement, perhaps degrading toward $2.2 \mathrm{GHz}$, which suggests there is little other extended emission in $\mathrm{Wd} 1$ beyond the regions demarked in Fig. 4.

Using the total fluxes above for the extended emission in $\mathrm{Wd} 1$ gives a spectral index of $-0.26 \pm 0.07$, consistent with optically-thin thermal emission. Following the prescription given in Sect. 4.3, an 8.6-GHz flux of $310 \mathrm{mJy}$ from a region $1.5^{\prime}$ in diameter leads to an ionized mass of $15 M_{\odot}$ for a plasma temperature of $10 \mathrm{kK}$, in excellent agreement with the ionized mass derived by Kothes \& Dougherty (2007).

C98 showed that the nebular radio emission associated with the RSG W26 correlated with mid-IR emission. Figure 4 shows an overlay of the radio emission in Wd 1 with Spitzer GLIMPSE data at $8 \mu \mathrm{m}$ (Benjamin et al. 2003). Without being distracted by the saturation and artefacts in the mid-IR image, it is striking that all the extended radio emission is associated with mid-IR emission. Additionally, note that mid-IR emission is associated with each of the RSG and YHG stars in the cluster (Sect. 4.3). Such a close correlation between the ionized emission evidenced in the radio and the $8 \mu \mathrm{m}$ emission is well established in ultra-compact HII regions, where the $8 \mu \mathrm{m}$ emission is due to excited PAH emission (e.g. Hoare et al. 2007) whereas in more evolved HII regions there is a clear association with hot dust, and the PAH emission associated with the photo-dissociation region beyond the ionized gas (e.g. Povich et al. 2007; Watson et al. 2008)

\section{The stellar sources}

The post-MS evolution of stars with masses in excess of $\sim 30 M_{\odot}$ is characterized by significant mass loss. While such mass loss plays a critical role in determining the subsequent evolution of the stars, the physical processes driving it are currently illconstrained, with the mass-loss rate and wind velocities anticipated to differ by several orders of magnitude depending on the exact evolutionary phase. Given that Wd 1 is uniquely well stocked with examples of every known post-MS stellar type, these observations offer the possibility of constraining this process.

Consequently, the following discussion on the nature of the radio emission for the 18 stellar detections is presented in a likely evolutionary sequence from OB supergiants, through cool super-/hypergiants, early hypergiants and WN and WC Wolf-Rayet stars. Given their comparatively weak winds $\left(\sim 10^{-7} M_{\odot} \mathrm{yr}^{-1}\right)$, the mid-to-late O-type main sequence progenitors of these stars are not detected (Martins et al. 2005). The emission associated with the $\mathrm{sgB}[\mathrm{e}]$ star W9 is discussed outside this evolutionary sequence, due to the uncertain placement of the $\mathrm{sgB}[\mathrm{e}]$ phase in the post-MS stellar zoo.

Throughout the discussion it will be assumed that $\mathrm{Wd} 1$ is at a distance of $4 \mathrm{kpc}$. Recent deep-IR imaging identifies the MS and pre-MS populations of $\mathrm{Wd} \mathrm{1,} \mathrm{leading} \mathrm{to} \mathrm{a} \mathrm{photometric} \mathrm{dis-}$ tance of $4.0 \pm 0.3 \mathrm{kpc}$ (Brandner et al. 2008), consistent with both $4.7 \pm 1.1 \mathrm{kpc}$ from an analysis of near-IR photometry of the WR stars in Wd 1 (Crowther et al. 2006), and on an atomic hydrogen absorption distance of 3.9 $\pm 0.7 \mathrm{kpc}$ (Kothes \& Dougherty 2007).

\subsection{W9 - a luminous radio source}

By far the brightest radio source in $\mathrm{Wd} 1$ is $\mathrm{W} 9$, with a total flux at $8.6 \mathrm{GHz}$ of $55.4 \mathrm{mJy}$, giving an $8.6-\mathrm{GHz}$ luminosity of $1.6 \times 10^{21} \mathrm{erg} \mathrm{s}^{-1}$. This places W9 among the most radio luminous thermal emitting stars known: a factor of a few less than the 8.6-GHz luminosity of the extreme LBV $\eta$ Carina $\left(4.5 \times 10^{21} \mathrm{erg} \mathrm{s}^{-1}\right)$ at radio minimum $(\sim 600 \mathrm{mJy}$; Duncan \& White 2002), comparable to the massive overcontact binary RY Scuti (Gehrz et al. 1995) and a factor of a few more luminous than the other oft-cited radio luminous thermal emitter MWC 349A (e.g. Tafoya et al. 2004).

C98 hypothesised that W9 comprised of two components, a compact stellar wind source and an extended region. The observations presented in this paper support this model, with an unresolved source surrounded by an extended region of emission that are both detected at all four observing frequencies.

The spectral index of the unresolved source coincident with W9 is $+0.68 \pm 0.08$, consistent with thermal emission arising from a partially optically thick, steady-state stellar wind with an $r^{-2}$ radial density distribution. It is assumed this component represents the present day stellar wind from W9. The spectral index of the extended region is $+0.16 \pm 0.07$, which we take to be essentially flat, and arguably consistent with optically-thin thermal emission. Assuming the extended region has a $r^{-2}$ radial ion density distribution, similar to the stellar wind, the lack of a 
A\&A 511, A58 (2010)
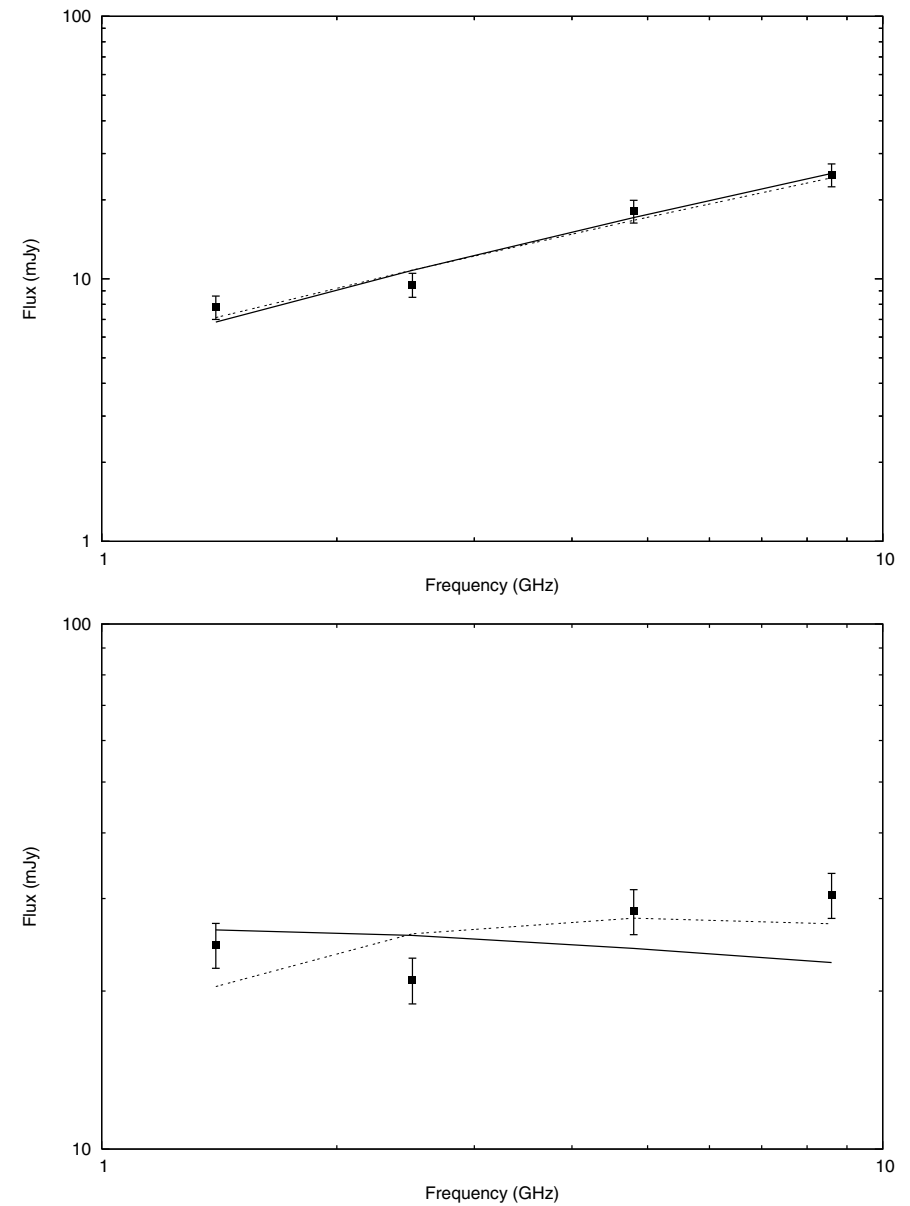

Fig. 5. Model fits to the continuum spectra of the unresolved partiallythick stellar wind (top) and extended, optically-thin (bottom) components of W9. The solid lines represent the best-fit model when the stellar wind outer radius/extended inner radii are constrained to be identical (Model 3). The dotted spectra are the best-fit models when the stellar wind outer radius and extended region inner radius are free parameters (Models 1 and 2).

turnover in its continuum spectrum to a positive spectral index implies that the extended region is totally optically thick down to $1.4 \mathrm{GHz}$. With the free-free opacity for an $r^{-2}$ ion distribution at frequency $v$ behaving as $\tau_{v}(p) \propto p^{-3} v^{-2.1}$ where $p$ is the impact parameter (see Eq. (A.2)), the lack of a turnover implies that the extended region must have an inner radius that is larger than the radius of the $\tau_{v}=1$ surface at $1.4 \mathrm{GHz}$. In this case, it is suggested that the extended region represents an earlier phase of mass-loss from W9, prior to the start of the current stellar wind phase.

To model the continuum spectra of both the stellar wind and the extended envelope a shell-like geometry is adopted, with outer and inner radii $R_{\mathrm{O}}$ and $R_{\mathrm{i}}$ respectively and with a $r^{-2}$ radial ion distribution (e.g. Taylor et al. 1987). The freefree opacity at radius $p$ in such an envelope is determined using Eqs. (A.2), (A.3) and (A.4). Together with Eq. (A.1), these lead to the flux from the envelope at frequency $v$. Assuming an electron temperature $T_{\mathrm{e}}=10 \mathrm{kK}$ and an outflow velocity of $200 \mathrm{~km} \mathrm{~s}^{-1}$, a best-fit of this model to the continuum spectra of the two components over the four observing frequencies gives the parameters of the two components (Table 5 and Fig. 5). The uncertainties in the fitting parameters are derived in the standard manner of fixing all but one parameter which is varied until $\chi^{2}$ changes by unity.
Table 5. Best-fit parameters of the $r^{-2}$ shell model applied to the continuum spectra of the compact and extended radio components of W9.

\begin{tabular}{lllll}
\hline \hline Model & Component & $\begin{array}{l}\dot{M} M_{\odot} \mathrm{yr}^{-1} \\
\left(\times 10^{-5}\right)\end{array}$ & $\begin{array}{l}R_{\mathrm{i}} \\
(\mathrm{AU})\end{array}$ & $\begin{array}{l}R_{\mathrm{o}} \\
(\mathrm{AU})\end{array}$ \\
\hline 1 & Compact & $8.7_{-0.2}^{+0.5}$ & 0 & $5800_{-1200}^{+7200}$ \\
2 & Extended & $17.6_{-0.4}^{+0.5}$ & $1100_{-90}^{+80}$ & $\infty$ \\
3 & Compact & $9.2_{-0.5}^{+0.4}$ & 0 & $4000_{-900}^{+3000}$ \\
3 & Extended & $33_{-5}^{+12}$ & $4000_{-900}^{+3000}$ & $\infty$ \\
\hline
\end{tabular}

Notes. Models 1 and 2 correspond to best-fits of each individual spectra. Model 3 assumes the outer radius of the compact component is equal to the inner radius of the extended component. For each model it is assumed that $T_{\mathrm{e}}=10 \mathrm{kK}, v_{\infty}=200 \mathrm{~km} \mathrm{~s}^{-1}, \mathrm{Z}=0.9, \mu=1.5, \gamma=0.8$ and the volume filling factor $f=1$.

A total of three models were examined. Model 1 leads to a mass-loss rate of $8.7_{-0.2}^{+0.5} \times 10^{-5}\left(v_{\infty} / 200 \mathrm{~km} \mathrm{~s}^{-1}\right) f^{1 / 2} M_{\odot} \mathrm{yr}^{-1}$ for $Z=0.9, \mu=1.5, \gamma=0.8, T=10 \mathrm{kK}$ (e.g. Leitherer $\&$ Robert 1991) for a volume filling factor for clumps $f$ (Abbott et al. 1981). The mass-loss rate is scaled to $200 \mathrm{~km} \mathrm{~s}^{-1}$ since the outflow velocity in W9 is not well constrained with observed line widths that range between 40 and $800 \mathrm{~km} \mathrm{~s}^{-1}$ (C08). Assuming the same outflow velocity and clump structure, the derived massloss rate of the extended region (Model 2) is approximately twice as high as the current phase of mass-loss, implying an earlier era of higher mass-loss rate $\sim 10^{-4} M_{\odot} \mathrm{yr}^{-1}$.

The model spectrum of the individual radio components established that the best-fit outer radius of the compact component (the current stellar wind) is greater than the inner bound of the extended region (earlier phase of mass-loss). If the massloss that produced both components is radially symmetric, it is not plausible for the inner radius of the extended region to be smaller than the extent of the current stellar wind. The third model (Model 3) assumed the inner bound of the extended region is at least as large as the outer bound of the current stellar wind. Fitting both continuum spectra simultaneously through minimizing the total $\chi^{2}$ from the two models gives the parameters of Model 3, which supports a factor of 3 higher mass-loss rate in the earlier phase of mass-loss than in the current stellar wind. The resulting model fits to the data are shown in Fig. 5.

The derived mass-loss rate for the putative earlier mass-loss phase is high for a massive star, being of the same order as the the current mass-loss rate in $\eta$ Carina of $\approx 10^{-4}-10^{-3} M_{\odot} \mathrm{yr}^{-1}$ (Corcoran 2008). The inner bound of $\sim 4000$ AU suggests that this mass-loss epoch would have ended $\sim 200 \mathrm{yr}$ ago assuming an outflow velocity of $200 \mathrm{~km} \mathrm{~s}^{-1}$, followed subsequently by the current mass-loss phase with $3 \times$ lower density.

With such a model for the circumstellar environment, what does this imply for the evolutionary status of W9? While veiling any photospheric features, the rich optical-IR emission line spectrum and IR excess indicate a rich circumstellar environment and permit a formal $\mathrm{sgB}[\mathrm{e}]$ star classification (C08; Ritchie et al. 2010). However spectral peculiarities exist when compared to other examples; most notably the unusual composite line profiles of He I 5876 and $6678 \AA$ A. The X-ray emission from W9 supports a binary system, being too hard $(k T \sim 3 \mathrm{keV})$ and bright $\left(L_{x} \sim 10^{33} \mathrm{erg} \mathrm{s}^{-1}\right)$ to arise in a single star, although it does not constrain the nature of the components (C08; Ritchie et al. 2010). Likewise, while W9 is found to be photometrically variable, no period has been identified currently (Bonanos 2007). Identification of W9 as a binary awaits further supporting observational evidence. 
The current mass-loss rate of W9 is approximately an order of magnitude higher than that inferred for any of the other transitional stars within Wd 1. It is directly comparable to those of the LBVs AFGL 2298, AG Car, FMM 362 and the Pistol star in quiescence with $\dot{M}=3-6 \times 10^{-5} f^{1 / 2} M_{\odot} \mathrm{yr}^{-1}$ (Clark et al. 2009; Groh et al. 2009; Najarro et al. 2009), despite being a factor of $\sim 2 \times$ less luminous ${ }^{1}$. Moreover, depending on the outflow velocity and wind-clumping factor the mass-loss rate for W9 is uncomfortably close to the limit expected for a line-driven wind for which Smith \& Owocki (2006) estimate a maximum massloss rate of

$$
1.4 \times 10^{-4}\left(L / 10^{6} L_{\odot}\right) M_{\odot} \mathrm{yr}^{-1} \text {. }
$$

Such a high mass-loss rate and the presence of a detached shell of material formed in a previous phase of enhanced mass loss is suggestive of an LBV identification, with $\dot{M} \sim 3 \times 10^{-4} M_{\odot} \mathrm{yr}^{-1}$ for W9 during this event matching the time averaged mass-loss rate inferred for the LBV Wra751 by Voors et al. (2000), as well as the YHG IRC+10 420 during the formation of its nebula (Sect. 4.3). The observed line spectrum, absolute visual magnitude and spectral energy distribution exclude the possibility that W9 is currently in a cool hypergiant phase with the binary companion providing the requisite ionizing flux to yield the emission line spectrum. Therefore, if the enhanced mass-loss rate is solely driven by the underlying star, this would be one of the few known examples of an LBV caught in a high mass-loss rate episode. Moreover, it would demonstrate that significantly enhanced mass loss in $\sim 40 M_{\odot}$ stars is not solely limited to occurring in a cool hypergiant phase.

However, while the current observational data are consistent with the ejecta being formed as a result of the post-MS evolution of a single massive star, its formation in an interacting binary system undergoing significant mass transfer, in turn leading to common envelope evolution, also appears viable and well motivated given the evidence of W9 being a binary. Examples of massive binaries with both a normal and a relativistic companion are known: RY Scuti and SS433 respectively, and thus provide observational templates. As with W9, both these systems have rich emission-line spectra and IR excesses due to the presence of circumstellar dust (Gehrz et al. 1995; Clark et al. 2007, and references therein). More interestingly, both are associated with compact, bright radio nebulae with sizes and fluxes directly comparable to W9 yet attributed to mass lost through binary interaction (Blundell et al. 2001; Gehrz et al. 1995). The former authors derive a mass-loss rate of the order of $10^{-4} M_{\odot} \mathrm{yr}^{-1}$ from the radio observations for SS433, while the latter infer an ionized mass of $10^{-3} M_{\odot}$ for the nebula associated with RY Scuti, with a mass-loss rate of $\sim 10^{-4}-10^{-5} M_{\odot} \mathrm{yr}^{-1}$ inferred from optical emission lines (de Martino et al. 1992); both rates are comparable with W9.

\subsection{The $O B$ supergiants}

Currently, there are $\sim 75$ spectroscopically identified evolved O stars (O giants and OB supergiants) within Wd 1, with a comparable number of stars classified as such on the basis of photometry (C05; C08; Negueruela et al. 2010) $)^{2}$. Unfortunately, the

\footnotetext{
1 In the absence of a bolometric correction for W9 adopting a comparable luminosity to the cool hypergiants - appropriate considering postMS evolution is expected to proceed at constant bolometric evolution prior to the WR phase - suggests $\log \left(L / L_{\odot}\right) \sim 5.8$.

2 Note that we include implicitly the mid-B supergiants such as W70, 71 and 57 here, while deferring discussion of the more luminous, and likely more evolved, B hypergiants W7, 13, 33 42a and 243 to Sect. 4.4.
}

currently published spectroscopy of these objects is of insufficient resolution and wavelength coverage to yield accurate physical parameters. However, canonical wind parameters for late O/early B supergiants (see C05, Table A2) indicate that at a distance of $\sim 4 \mathrm{kpc}$ thermal wind emission would be undetectable, due to a combination of comparatively high terminal wind velocity and low mass-loss rate. As an example, the O9.5 Ia star with $v_{\infty} \sim 1900 \mathrm{~km} \mathrm{~s}^{-1}$ and $\dot{M}=6 \times 10^{-6} M_{\odot} \mathrm{yr}^{-1}$ and adopting $Z=\gamma=1$ and $\mu=1.34$ (Leitherer et al. 1995) has an expected 8.6-GHz flux of $\sim 0.06 \mathrm{mJy}$, much lower than the $8.6-\mathrm{GHz}$ detection limit of $0.17 \mathrm{mJy}$. Since radio flux is $\propto f^{-3 / 2}$, it would take a highly clumpy wind with $f \sim 0.5$ to make such a wind detectable.

Consequently, the apparent detections of W15 and W17 (O9Ib and O9Iab respectively - Negueruela et al., in prep.), D09-R1 and potentially D09-R2 (both B0I or earlier; C08) are somewhat unexpected, most especially considering that these stars are unremarkable compared with the rest of the OB stars studies spectroscopically. Of note is that W15, W17 and D09$\mathrm{R} 1$ all are coincident with extended emission regions and the parameters of the unresolved radio components coincident with the underlying stars are likely influenced by this extended emission. As a result, the derived parameters may not reflect the intrinsic properties of the underlying stars, but rather the extended plasma region.

That withstanding, W17 has a spectral index marginally steeper than -0.1 , hinting at non-thermal emission and a potential CWB. However, we note it does not have detected X-ray emission $\mathrm{C} 08$ and shows no evidence of RV variations (Ritchie et al. 2009a). W15 has a spectral index lower limit of 0.0, and it is not possible to distinguish whether the emission is from either a stellar wind or a non-thermal/thermal composite source. It shows no evidence for binarity in either RV observations (Ritchie et al. 2009a) or X-ray observations (C08), where the emission is soft $(k T \sim 0.6 \mathrm{keV})$ and at a level expected for a single O-type star $\left(\sim 2 \times 10^{32} \mathrm{erg} \mathrm{s}^{-1}\right.$; e.g. Berghoefer et al. 1997) as a result of shock heating within the stellar wind. Nevertheless, if the radio flux is attributed to thermal emission from a stellar wind, W15 would have an unusually high mass-loss rate of $\dot{M}=3 \times 10^{-5} M_{\odot} \mathrm{yr}^{-1}$ in comparison to other late-O/early-B supergiants (Crowther et al. 2006; Mokiem et al. 2007). The absence of RV variations in these stars does not rule out a binary interpretation unequivocally since the mass ratios may be extreme and the inclination of the orbit unfavorable for ready detection of RV variations. Furthermore, the orbital period could be significantly in excess of the observation timescale.

The spectral index of the point source associated with D09-R1 is complicated by the presence of an extended component, and given the absence of both optical spectroscopy and an $\mathrm{X}$-ray detection, it is simply concluded that the nature of this emission remains uncertain, and note that if it is assumed to be a stellar wind, the same problems with respect to an extreme mass-loss rate as found for W15 are encountered.

\subsection{The cool super- and hypergiants}

All four of the RSGs and at least four of the six YHGs in Wd 1 are radio sources. Irrespective of the uncertain identification of W16a as a radio emitter, the fraction of radio sources amongst both these classes of object is high. Of the RSGs, W75 was the weakest source detected and was unresolved at any wavelength. The other three detected RSGs (W20, 26 and 237) have extended radio emission. 
For both W20 and W26 the radio emission is best characterised by two components: a compact $\left(\sim 2^{\prime \prime}\right)$, spatially resolved source coincident with the star, and a more extended nebula $\left(>10^{\prime \prime}\right)$. W237 is similar in structure though the inner component coincident with the star is unresolved. In W20 and W26 the more extended components have a distinct cometary morphology that extends away from the central region of the cluster, while that in W237 is more elliptical.

The radio properties of the YHGs are more heterogeneous. W8 is not detected. W32 is associated with a weak point source, while W12 and W265 are associated with compact resolved sources, similar to the RSG radio sources. Finally, the radio emission from W4 consists of a point source at the position of the star surrounded by an extended component.

The majority of the cool super- and hypergiants have spectral indices that are flat, within the uncertainties. Unlike OB and WR stars, composite thermal and non-thermal spectra have not been identified previously, and are not expected. Hence we identify these indices with optically-thin thermal emission. For a Maxwellian plasma at temperature $T$, the optically-thin radio flux density is given by

$S_{v}=5.7 \times 10^{-56} T^{1 / 2} g_{v} D^{-2} E_{V} \mathrm{mJy}$,

where $D$ is the distance in kpc and $E_{V}$ is the emission integral

$E_{V}=\int n_{\mathrm{e}}^{2} \mathrm{~d} V=6 \times 10^{57}\left(\frac{D}{4 \mathrm{kpc}}\right)^{2}\left(\frac{S_{8.6}}{1 \mathrm{mJy}}\right)\left(\frac{T}{10 \mathrm{kK}}\right)^{-\frac{1}{2}} \mathrm{~cm}^{-3}$

at $8.6 \mathrm{GHz}$. The total ionized mass can then be determined from $M_{T}=\mu m_{\mathrm{H}}\left(E_{V} / n_{\mathrm{e}}\right)$.

The ionized masses of the nebulae, based on the source parameters determined at $8.6 \mathrm{GHz}$, are given in Table 6 . Note this is not the total mass since these observations are insensitive to any neutral component. On average, the emission integral $E_{V} \sim 1.5 \times 10^{58} \mathrm{~cm}^{-3}$, implies an ionizing luminosity that has to be greater than $E_{V} \alpha^{(2)} \sim 6 \times 10^{45} \mathrm{~s}^{-1}$, where $\alpha^{(2)}$ is the is the hydrogen recombination rate to all but the ground state. Such ionizing luminosity is not available from these relatively cool stars with stellar effective temperatures $\leq 5000 \mathrm{~K}$ (e.g. de Jager 1998).

How are these envelopes ionized? A cluster of mass $10^{5} M_{\odot}$ and solar metallicity can easily provide such a photon luminosity and seems the most likely source of the ionizing radiation. Taking the cluster to be approximately $1 \mathrm{pc}$ across and with 100 OB-type stars implies that on average each OB star occupies a volume $0.04 \mathrm{pc}^{3}$, and is separated from the next OB star by $\sim 0.34 \mathrm{pc}$. If each OB star provides $10^{48} \mathrm{~s}^{-1}$ ionizing photons (Martins et al. 2005), with $\sim 10^{45} \mathrm{~s}^{-1}$ required to fully ionize the cool hypergiant envelopes, suggests a radiation dilution factor $\propto\left(R_{*} / D\right)^{-2}$ less than 1000 , which implies the hypergiant envelopes are $\sim 0.01 \mathrm{pc}$ radius. This is closely consistent with the sizes observed for RSG nebulae e.g. VY CMa (see Sect. 4.3.2).

Alternatively, a hot companion star could provide the required photon flux, which at $10^{45-46} \mathrm{~s}^{-1}$ implies an early Btype star. The YHG HR 8752 provides a precedent for such a model: it is detected at radio wavelengths and together with [N II]-emission lines, needs a source of ionizing radiation that is provided by a putative B1 companion (Stickland \& Harmer 1978). In this respect we highlight the presence of [N II]emission lines in the optical spectra of W20, W237 and W265 (Clark et al. 2010).

If the plasma in these optically-thin nebulae is due to mass loss from the underlying stars, having optically-thin spectra
Table 6. Ionized mass estimates for the optically-thin stellar nebulae around the YHGs (top panel) and RSGs (bottom panel) in Wd 1.

\begin{tabular}{llllllll}
\hline \hline ID & $S_{8.6}$ & Maj & $\frac{\text { Min }}{\text { Major }}$ & $\begin{array}{l}\text { Vol } \\
10^{50} \\
\left(\mathrm{~cm}^{3}\right)\end{array}$ & $\begin{array}{l}E_{V} \\
10^{58} \\
\left(\mathrm{~cm}^{-3}\right)\end{array}$ & $\begin{array}{l}n_{\mathrm{e}} \\
10^{3} \\
\left(\mathrm{~cm}^{-3}\right)\end{array}$ & $\begin{array}{l}\text { Mass } \\
10^{-3} \\
\left(M_{\odot}\right)\end{array}$ \\
\hline 4 & 2.2 & 2.45 & 0.49 & 4.0 & 1.3 & 5.7 & 2.9 \\
$12 \mathrm{a}$ & 2.9 & 1.82 & 0.92 & 5.7 & 1.7 & 5.5 & 3.9 \\
265 & 2.3 & 2.86 & 0.54 & 7.7 & 1.4 & 4.2 & 4.1 \\
\hline 20 & 15.6 & 13.79 & 0.48 & 677.9 & 9.2 & 1.2 & 99.6 \\
26 & 173.4 & 15.0 & 0.33 & 412.4 & 102.2 & 5.0 & 259.0 \\
237 & 5.6 & 11.19 & 0.76 & 908.0 & 3.3 & 0.6 & 69.1 \\
\hline
\end{tabular}

Notes. A distance of $4 \mathrm{kpc}$ is assumed.

down to a frequency of $1.4 \mathrm{GHz}$ implies that the ionized extended envelopes have inner radii significantly larger than the stellar radii. If this is the case, two possible models for the envelope can be advanced. The mass loss from these objects could have stopped or significantly decreased at some point in the past resulting in a detached shell. Alternatively, the ionized region may only occupy the periphery of the envelope, with the interior part of the envelope being neutral. Such a geometry could result from external ionization from the cluster radiation field. High-resolution observations are required to substantiate either of these potential models.

\subsubsection{The yellow hypergiants}

Given the rarity of YHGs and the difficulty identifying suitable observational diagnostics, few measurements of the quiescent or outburst wind velocities and mass-loss rates of YHGs exist in the literature. For HD $33579\left(\mathrm{~A}_{3} \mathrm{Ia}^{+}\right)$in the LMC, Stahl et al. (1991) quote $v_{\infty} \sim 200 \mathrm{~km} \mathrm{~s}^{-1}$ and $\dot{M} \sim 2 \times 10^{-6} M_{\odot} \mathrm{yr}^{-1}$, and Israelian et al. (1999) report $v_{\infty} \sim 120 \mathrm{~km} \mathrm{~s}^{-1}$ and $\dot{M}<$ $6.7 \times 10^{-6} M_{\odot} \mathrm{yr}^{-1}$ for HR 8752 in 1998 . For the well-known example $\rho$ Cas, Lobel et al. (1998) find $v_{\infty} \sim 120 \mathrm{~km} \mathrm{~s}^{-1}$ and $\dot{M}<9.2 \times 10^{-5} M_{\odot} \mathrm{yr}^{-1}$ in 1993 , in contrast to $v_{\infty} \sim 35 \mathrm{~km} \mathrm{~s}^{-1}$ and $\dot{M} \sim 5.4 \times 10^{-2} M_{\odot} \mathrm{yr}^{-1}$ during the 2000 outburst (Lobel et al. 2003). Castro-Carrizo et al. (2007) measure outflow velocities of $35 \mathrm{~km} \mathrm{~s}^{-1}$ and $25-37 \mathrm{~km} \mathrm{~s}^{-1}$ for the ejecta associated with HD 179821 and IRC +10420 (although a priori it is not clear if they were ejected in a RSG or YHG phase). For both these stars, variable mass-loss rates of $4-300 \times 10^{-5} M_{\odot} \mathrm{yr}^{-1}$ and $1.2-3 \times 10^{-4} M_{\odot} \mathrm{yr}^{-1}$ were found respectively during the nebulae formation events, with Blöcker et al. (1999) also reporting a mass-loss rate in excess of $10^{-3} M_{\odot} \mathrm{yr}^{-1}$ for IRC +10420 in the recent past. These works all suggest that during outburst the mass-loss rates in YHGs are higher than during quiescence whereas the outflow velocity is lower during outburst.

Taking a mean 8.6-GHz flux of $2.5 \mathrm{mJy}$ for the YHGs, their radio luminosity is $4.9 \times 10^{19} \mathrm{erg} \mathrm{s}^{-1}$. The only other radio detected YHG in the Galaxy, HR 8752, has an $8.6-\mathrm{GHz}$ flux of $17 \mathrm{mJy}$ (Dougherty et al., in prep.) at a distance of $1.7 \mathrm{kpc}$, giving a luminosity of $5.9 \times 10^{19} \mathrm{erg} \mathrm{s}^{-1}$, similar to the YHGs in Wd 1. HR 8752 has been interpreted to have a stellar wind from measured spectral indices of $0.51 \pm 0.08$ (Higgs et al. 1978) and $0.61 \pm 0.04$ (Dougherty et al., in prep.). Only the compact component in W4 appears to have a spectral index consistent with a stellar wind $(+0.49 \pm 0.31)$, but given the uncertainty, arguably also consistent with optically-thin thermal emission. Assuming $Z=0.9, \mu=1.5$ and $\gamma=0.8$ as for W9 and that the putative wind from W4 is fully ionized and smooth $(f=1)$ gives a mass- 
loss rate of $0.7 \times 10^{-5}\left(v_{\infty} / 200 \mathrm{~km} \mathrm{~s}^{-1}\right) M_{\odot} \mathrm{yr}^{-1}$. This is consistent within a factor of a few with the quiescent mass-loss rates of HD 33579, HR 8752 and $\rho$ Cas, but significantly lower than inferred for $\rho$ Cas, HD179821 and IRC +10 420 during outburst, especially for comparably low velocities $\left(<40 \mathrm{~km} \mathrm{~s}^{-1}\right)$.

The extended nebulae around W4, W12a and W265 have masses of $3-4 \times 10^{-3} M_{\odot}$ and radii of $\sim 0.02 \mathrm{pc}$ (based on the major axis size in Table 6). An assumed outflow velocity of $\sim 200 \mathrm{~km} \mathrm{~s}^{-1}$ gives a flow time of $\sim 100 \mathrm{yr}$, during which time mass loss at a rate of $\sim 10^{-5} M_{\odot} \mathrm{yr}^{-1}$, as found for $\mathrm{W} 4$ above, yields a total mass loss of $10^{-3} M_{\odot}$. Thus, by comparison with the cases of $\rho$ Cas, IRC +10420 and HR 8752, the radio nebulae associated with the YHGs in $\mathrm{Wd} 1$ may be the result of quiescent mass loss, rather than an outburst phase.

None of the YHGs in Wd 1 possess the old ( 4000 yr), massive $\left(M>1 M_{\odot}\right)$ ejection nebulae seen in HD 179821 and IRC +10 420 (Oudmaijer et al. 1996; Castro-Carrizo et al. 2007). We suspect that in the extreme environment of Wd 1 such nebulae would quickly be ablated by the cluster wind arising from the OB stars - as appears to be the case for the RSGs W20 and W26 (see Sect. 4.3.2). However we cannot exclude the possibility that the YHGs may be evolving to cooler temperatures (redwards) and have yet to encounter the instabilities leading to extreme mass-loss rates in either the RSG or post-RSG phase. A mixture of both pre- and post-RSG objects combined with the intrinsic variability of YHGs (Clark et al. 2010) may explain the heterogeneous radio properties of the $\mathrm{Wd} 1$ population. This argument is supported by evidence that even the best two candidates for post-RSG stars, HD 179821 and IRC +10 420, exhibit quite dissimilar nebular properties (Oudmaijer et al. 2009; Patel et al. 2008).

\subsubsection{The red supergiants}

Given very high mass-loss rates inferred for extremely luminous RSGs $\left(\log \left(L / L_{\odot}\right)>5.5\right)$, it is suspected that they play an important role in post-MS evolution of stars at the HumphreysDavidson limit. Consequently, they have been the subject of numerous (multi-wavelength) studies to constrain their mass-loss histories. Of the Galactic examples, Schuster et al. (2006) used the HST/WFPC2 to identify compact ( $r \leq 0.003 \mathrm{pc})$ nebulae associated with NML Cyg, VX Sgr and S Per, with Morris \& Jura (1983) also associating a much larger (>1 pc) H II region with NML Cyg. de Wit et al. (2008) report the presence a nebula around $\mu$ Cep at 25 microns $(r \sim 0.03 \mathrm{pc})$; a comparably sized nebulae surrounds VY CMa $(r \sim 0.04 \mathrm{pc}$ in HST/WFPC2 images; Smith et al. 2001). Finally Serabyn et al. (1991) and Yusef-Zadeh \& Morris (1991) report the presence of an asymmetric nebula ( $\sim 1 \mathrm{pc}$ in length) around the Galactic Centre RSG IRS7 at both near-IR and radio wavelengths.

As with these stars, the discovery of large nebulae associated with three of the 4 RSGs in Wd 1 (major axis $>0.2 \mathrm{pc}$; Table 6) emphasises the role of mass loss in this evolutionary stage. NML Cyg and IRS7 are of special interest in the context of the RSGs in $\mathrm{Wd} \mathrm{1,} \mathrm{given} \mathrm{that} \mathrm{these} \mathrm{stars} \mathrm{are} \mathrm{also} \mathrm{located} \mathrm{within} \mathrm{dense}$ stellar aggregates, namely Cyg OB2 (Morris \& Jura 1983) and the Galactic Centre cluster (e.g. Yusef-Zadeh \& Morris 1991) respectively. Indeed the pronounced cometary nebulae of W20 and W26 appear similar both in morphology and linear scale to that of IRS7. Consequently we attribute the shape of these nebulae to a similar physical process, namely the interaction of the ejecta/RSG stellar wind with the cluster medium/wind. Lacking dynamical information, we refrain from attempting a quantitative analysis of the nebular morphologies, but note that the unremarkable radial velocities found for these stars by Mengel $\&$ Tacconi-Garman (2007) suggest that the dominant cause of ram pressure shaping the nebulae is the expansion of the cluster wind, rather than the rapid motion of the stars through a quasistatic cluster medium. This is supported by the orientation of each of these cometary nebulae away from the cluster centre region.

The morphology of the radio nebula associated with W237 suggests it has been less affected by the cluster wind than either W20 or W26. Adopting an outflow velocity of $30 \mathrm{~km} \mathrm{~s}^{-1}$ for the RSG wind, the mass of $0.07 M_{\odot}$ and radius of $\sim 0.11 \mathrm{pc}$ (Table 6) results in a kinematic age of $3600 \mathrm{yr}$ and a time averaged mass-loss rate of $2 \times 10^{-5} M_{\odot} \mathrm{yr}^{-1}$. This age supports the suggestion that the radio nebula is little affected by the cluster wind, otherwise it likely would be ablated as observed in $\mathrm{W} 20$ and W26. This is consistent with its position further from the cluster centre than either W20 or W26. The derived massloss rate is comparable to the mass-loss rates found for field RSGs, which range from $10^{-6}-10^{-4} M_{\odot} \mathrm{yr}^{-1}$ (Jura \& Kleinmann 1990; Sylvester et al. 1998), although transient rates as high as $\sim 10^{-3} M_{\odot} \mathrm{yr}^{-1}$ are seen in NML Cyg and VY Cma (Blöcker et al. 2001; Smith et al. 2001) and as low as a $\sim$ few $10^{-7} M_{\odot}$ in $\mu$ Cep (de Wit et al. 2008) have been observed.

Moreover, both W20 and W26 have similar nebular masses to W237 and, in turn, all three are directly comparable to that of the nebula surrounding VY CMa (Smith et al. 2001). Consequently the ejecta associated with the RSGs within Wd 1 appear to be the result of similarly extreme, possibly transient mass-loss events as those that yield the nebulae around other Galactic RSGs (e.g. Smith et al. 2001). In this respect, the apparent instabilities in W26 and W237 are of interest (Clark et al. 2010).

\subsection{The $B$ hypergiants}

The final subset of transitional stars to be considered are the B hypergiants, which likely represent the immediate progenitors of WN-type stars. Included in this group are the LBV W243 and the cool B-type hypergiants W7, 33 and 42 . The final member is the early-B hypergiant W13, which emphasises the close physical continuity between the B hypergiants and the hydrogen-rich late-WN stars such as W44 (=WR L (WN9h); C08) detected at 8.6 GHz (Table 2 and Sect. 4.5). Of these stars, only W243 has a spectral index indicative of thermal wind emission.

W243 has recently been the subject of an extensive spectroscopic study by Ritchie et al. (2009b). This confirmed the finding of C02 that W243 had substantially cooled since the previous observations (e.g. Westerlund 1987), with the stellar temperature between $2002-8$ being $8-9 \mathrm{kK}$, with a formal spectral classification of mid-A type and a photospheric absorption spectrum of a YHG. However, detailed non-LTE analysis were unable to simultaneously reproduce these features and the prominent $\mathrm{H}$ I and He I emission lines, leading Ritchie et al. (2009b) to conclude that W243 harbored a hot binary companion. Unfortunately, current spectroscopic data are unable to constrain the nature of the hot companion; nevertheless at this time W243 closely resembles the known YHG binary HR8752 (Sect. 4.3.1) and we assume that the hot companion is responsible for the ionization of its wind.

Given the current properties of W243 we adopt identical values of $Z, \mu$ and $\gamma$ as used for the YHG W4, leading to a massloss rate of $1.1 \times 10^{-5}\left(v_{\infty} / 200 \mathrm{~km} \mathrm{~s}^{-1}\right) f^{1 / 2} M_{\odot} \mathrm{yr}^{-1}$, which is directly comparable to W4. This is to be expected considering their similar radio fluxes (Table 2). This mass-loss rate is an 
order of magnitude higher than that found by Ritchie et al. (2009b), correcting for difference in assumed outflow velocity, though it should be noted that the observed optical spectrum is relatively insensitive to the current mass-loss rate. Assuming a $4 \sigma$-detection limit of $0.24 \mathrm{mJy}$ for the remaining stars yields a corresponding upper limit to the mass-loss rates of $2 \times 10^{-6}\left(v_{\infty} / 200 \mathrm{~km} \mathrm{~s}^{-1}\right) M_{\odot} \mathrm{yr}^{-1}$.

In comparison, the LBVs HR Car and HD 160529 have mass-loss rates of $\sim 1-2 \times 10^{-5} f^{1 / 2} M_{\odot} \mathrm{yr}^{-1}$ determined from radio observations, with HD 80077 and HD 168607 an order of magnitude lower (Leitherer et al. 1995). Therefore, we conclude that the mass-loss behavior of the $\mathrm{Wd} 1$ population of $\mathrm{B}$ hypergiants is consistent with expectations drawn from the wider Galactic population. No extended emission is associated with any of these stars, whereas a number of Galactic LBVs are associated with radio bright nebulae. However such nebulae are typically found to be old $\left(\sim 10^{3}-10^{4}\right.$ yr; Clark et al. 2003) and of an extent sufficient to encompass the central regions of $\mathrm{Wd} 1$. As argued for the YHGs, we suspect that they would quickly be disrupted by the cluster wind and/or radiation field.

\subsection{Wolf-Rayet stars}

In recent years, Wd 1 has been shown to harbour at least 24 WR stars (Crowther et al. 2006), representing 8\% of the currently known galactic population. Five of these have been detected in this radio survey at a flux level typically $0.3-0.5 \mathrm{mJy}$ at 8.6 GHz: WR A (=W72), B, F(=W239), L(=W44), and $\mathrm{V}(=\mathrm{W} 31 \mathrm{~b})$ (Crowther et al. 2006, and references therein), potentially consistent with the radio emission originating in their stellar winds.

WR B is an exception, with a deduced flux of $4.3 \mathrm{mJy}$. This flux is $5-10 \times$ higher than the other detected WR stars, implying a wind density that is $3-6 \times$ higher than the mean value for the other WRs. Bonanos (2007) found that WR B is an eclipsing binary and in binary systems the flux can be raised substantially above that from a stellar wind by emission arising in a windcollision region (WCR) from the colliding winds of two massive stars in a binary (e.g Dougherty et al. 2005). More likely, the flux of WR B is biased by being a part of a more extended emission region (A3; Sect. 3.3), a suggestion supported by data reduction that indicates the $8.6-\mathrm{GHz}$ emission is resolved which is not expected for typical WR winds at this distance. For comparison, the closest WR star to the Sun, WR 11 at $\sim 0.26 \mathrm{kpc}$ (van der Hucht et al. 1997) has a stellar wind that is resolved by the ATCA at $8.6 \mathrm{GHz}$, with a size of 0.47 arcsec (Dougherty et al., in prep.). If WR 11 was at a distance of $4 \mathrm{kpc}$, the wind would be $\sim 30$ mas in diameter and not resolved. This leads us to believe that the source parameters derived for WR B are influenced by its location in the extended emission region A3.

W44 (WR L) is the only WR star that has a spectral index consistent with a partially optically-thick stellar wind, although the lower limit to the spectral indices of WR F leave this possibility open. For the WNVLh star WR L we adopt $\mu=2.0$, $Z=1.0$ and $\gamma=1.0$ (e.g. Leitherer et al. 1997), resulting in $\dot{M}=2 \times 10^{-5}\left(v_{\infty} / 1000 \mathrm{~km} \mathrm{~s}^{-1}\right) f^{1 / 2} M_{\odot} \mathrm{yr}^{-1}$ respectively. For the WC9 star WR F adopting $\mu=4.7, Z=1.1$ and $\gamma=1.1$ yields $\dot{M}=3.3 \times 10^{-5}\left(v_{\infty} / 1000 \mathrm{~km} \mathrm{~s}^{-1}\right) f^{1 / 2} M_{\odot} \mathrm{yr}^{-1}$. Both these mass-loss rates are comparable to those found for similar stars in the Galactic Centre cluster by Martins et al. (2007) and other field WRs (e.g. Cappa et al. 2004).

The remaining three WR detections (WR A, F, V) have flat, though highly uncertain, spectral indices. If they are flat, an interpretation due to composite spectra composed of both thermal and non-thermal emission components would be consistent with that observed in a number of $\mathrm{WR}+\mathrm{OB}$ binaries (Dougherty \& Williams 2000). Certainly, among WR stars with flat radio spectra, those that have been observed at high resolution (e.g. Williams et al. 1997; Dougherty \& Williams 2000; Dougherty et al. 2005, and references therein), all have identified WCRs arising in CWBs.

Recently, Wd 1 has been observed by CHANDRA (Clark et al. 2007; Skinner et al. 2006; Muno et al. 2006a,b) and the WR stars WR A, B, F and L are all X-ray bright $\left(L_{x} \sim 10^{32-33} \mathrm{erg} \mathrm{s}^{-1}\right)$ and hard $\left(k T>2.6 \mathrm{keV}\right.$ i.e. $\left.T>3 \times 10^{7} \mathrm{~K}\right)$. These temperatures are expected for plasma heating by shocks in a WCR (Stevens et al. 1992; Pittard 2009; Pittard \& Parkin 2009), which led both Clark et al. (2007) and Skinner et al. (2006) to suggest these WR systems are all CWB systems. In support of this argument, each of these WR stars have exhibited photometric variations in the optical (Bonanos 2007). WR B demonstrates clear eclipses while WR A demonstrates a 7.63-day periodic modulation to its light curve and is also found to be a spectroscopic binary (Crowther et al. 2006), though such a short-period system is not anticipated to exhibit evidence of non-thermal radio emission due to a wind collision (Dougherty \& Williams 2000). However, if all these X-ray bright WR stars are interpreted as CWBs, then the apparently thermal radio spectrum of WR L would be attributed to a sufficiently short-period binary where any non-thermal radio emission from a WCR is completely absorbed by the circumbinary stellar wind(s) or the relativistic electrons are cooled by the intense UV radiation of a putative massive companion.

The IR excess associated with WR F has been attributed to hot dust (Crowther et al. 2006; Groh et al. 2006), again supporting a CWB interpretation, since dusty WC stars are widely considered to be in binaries with $\mathrm{OB}$ companions where windwind interaction is the essential mechanism that attains the high gas densities required for dust formation (e.g. Williams 1999; Tuthill et al. 1999; Monnier et al. 1999; Williams et al. 2009)

A synthesis of the IR, X-ray and these radio observations therefore argues for a high binary fraction amongst the WR stars, with C08 suggesting that at least $70 \%$, and possibly the complete population, of WR stars in Wd 1 are in massive binary systems. However, given the lack of radial velocity variations from spectral line observations this assertion has yet to be verified, and may be exceedingly challenging to verify.

\section{Extended emission}

Lastly, we consider briefly the extended emission and its origin. Trivially, optically-thin thermal emission is associated with young massive clusters still embedded in Giant H II regions, where the young massive stars ionize the remnants of the natal Giant Molecular Cloud. However, this seems unlikely for Wd 1. At an age of $\sim 5 \mathrm{Myr}$ one might expect the action of a cluster wind to have sculpted a cavity $\sim 10$ parsec in extent e.g. the G305 star forming complex surrounding the young ( $\geq 2-5 \mathrm{Myr}$ ) massive clusters Danks 1 and Danks 2; Clark \& Porter (2004), while the optically-thin thermal clumps associated with $\mathrm{Wd} 1$ are found significantly closer to, and indeed within the confines of, the cluster. Kothes \& Dougherty (2007) report the presence of a large (25 pc radius) H I bubble (their feature B3) in the neutral environment of $\mathrm{Wd} 1$, which they attribute to the action of the cluster wind on the natal GMC and wider ISM. If the cluster wind swept the region clear of the natal material, the diffuse emission would then have to arise from material ejected recently from the stellar population of $\mathrm{Wd} \mathrm{1,} \mathrm{either} \mathrm{via} \mathrm{stellar} \mathrm{winds}$ or SNe. Assuming velocities of $20-200 \mathrm{~km} \mathrm{~s}^{-1}$ (appropriate 
for $\mathrm{RSG} / \mathrm{LBV}$ and $\mathrm{SNe}$ ejecta) at a displacement of a parsec yields an age for material of $5 \times 10^{3-4}$ yr. Furthermore, a total ionized mass of $15 M_{\odot}$ only requires an average mass-loss rate of a $3 \times 10^{-6} M_{\odot} \mathrm{yr}^{-1}$ over the cluster lifetime, readily available from the population in $\mathrm{Wd} 1$ as discussed in the previous sections.

An origin in recent stellar ejecta seems to be certain for stars such as W20 and W26 with their large cometary nebula, but may not be so clear for other regions such as A6, where no associated stellar source is readily apparent in the FORS2 image. The ionized mass of A6 is estimated to be $\sim 0.5 M_{\odot}$, which could have certainly originated from a stellar wind. In addition, A6 does have a cometary-like structure that also appears to point towards the cluster centre region, like the nebulae around W20 and W26 (see Fig. 4). An alternative explanation for some of the diffuse emission is that it arises in natal material left behind when the cluster region was largely swept clear by the cluster wind, possibly through shielding by over-dense regions in the natal cloud. The nature and origins of the extended emission will be explored further in a forthcoming paper.

\section{Summary}

We have presented the results of a multi-frequency radio survey of the galactic Super Star Cluster Wd 1, and used optical, IR and X-ray observations from the literature to elucidate the nature of the radio sources detected. We detect 18 radio sources for which positional coincidence suggests an association with a cluster member. These radio stars are associated with every class of post-MS star present in the cluster. Moreover, they comprise a diverse population of point-like, unresolved sources and extended, resolved sources, with spectral indices corresponding to thermal, non-thermal and composite thermal and non-thermal emission. Nevertheless, it appears possible to understand these properties under the current evolutionary paradigm for massive (binary) stars. In brief, the radio observations reveal the following properties for the massive stars in $\mathrm{Wd} 1$ :

- The brightest stellar radio source in Wd 1 is W9, with an 8.6-GHz luminosity that places it amongst the most luminous radio stars known. The emission comprises both a point source and a more extended nebula. The flux and spectral index of the point source implies an origin in a powerful stellar wind, with a mass-loss rate of $9.2_{-0.5}^{+0.4} \times$ $10^{-5}\left(v_{\infty} / 200 \mathrm{~km} \mathrm{~s}^{-1}\right) f^{1 / 2} M_{\odot} \mathrm{yr}^{-1}$. The extended nebula is deduced to arise from an earlier mass-loss epoch with a mass-loss rate $\sim 3$ times higher than the current stellar wind, close to the limit expected for a line-driven wind (Smith \& Owocki 2006). An obvious comparison to make is to LBVs, with W9 having a current mass-loss rate similar to galactic examples e.g. the Pistol star. Likewise, the mass-loss rate deduced for the extended nebula is comparable to several other galactic LBV's during outburst, although orders of magnitude less than inferred for both P Cygni and $\eta$ Carinae during outburst (Clark et al. 2009, and references therein). The $\mathrm{X}$-ray properties of W9 imply that it is a binary system, although it is not currently possible to constrain the nature of the companion. An alternative scenario with significant mass loss in a common envelope phase of stellar evolution cannot be excluded.

- Surprisingly, three of the $>100$ evolved OB stars (luminosity classes III-Ia) are detected, with radio fluxes an order of magnitude larger than expected for stellar winds in these types.
It is suggested they may be CWBs based on potentially composite spectra of both non-thermal and thermal emission. However, none of the three detected stars exhibit X-ray emission characteristic of CWBs or evidence of RV variations due to a binary companion. Alternatively, their seemingly high radio luminosity may be due the influence of the extended emission in which they are embedded.

- All four RSGs are detected, with three associated with large nebulae with ionized masses up to $\sim 0.26 M_{\odot}$, emphasising the importance of mass loss in this evolutionary phase. Of these, the nebulae around W20 and W26 have a pronounced cometary morphology, suggesting significant interaction with either the intracluster medium or cluster wind. W237 shows less evidence for such interaction and has a kinematic age of $\sim 3600 \mathrm{yr}$ and a time averaged mass-loss rate of $2 \times 10^{-5}\left(v_{\infty} / 30 \mathrm{~km} \mathrm{~s}^{-1}\right) M_{\odot} \mathrm{yr}^{-1}$. This is consistent with other field RSGs, although it is substantially lower than inferred for NML Cyg and VY CMa during the formation of their nebulae.

- The YHG W4 is argued to have a stellar wind with a massloss rate of $10^{-5}\left(v_{\infty} / 200 \mathrm{~km} \mathrm{~s}^{-1}\right) f^{1 / 2} M_{\odot} \mathrm{yr}^{-1}$, consistent with the few estimates available for other field YHGs. The extended nebulae associated with W4, 12a and 265 are significantly less massive than those associated with the RSGs in $\mathrm{Wd} \mathrm{1.} \mathrm{It} \mathrm{is} \mathrm{argued} \mathrm{they} \mathrm{arise} \mathrm{from} \mathrm{quiescent} \mathrm{mass} \mathrm{loss}$ rather than during outburst episodes.

- Since neither the YHGs nor RSGs are hot enough to ionize their own stellar winds and/or more extended nebulae, the requisite ionizing photons must arise from either an unseen companion or the cluster radiation field.

- Of the extreme B-type hypergiants, only the LBV W243 was detected, with a spectral index consistent with thermal emission. The corresponding mass-loss rate is directly comparable to that found for the YHG W4, as expected given the similarity in current spectral type and radio flux. Upper limits of $2 \times 10^{-6}\left(v_{\infty} / 200 \mathrm{~km} \mathrm{~s}^{-1}\right) f^{1 / 2} M_{\odot} \mathrm{yr}^{-1}$ for the three other $\mathrm{B}$ hypergiants were found, consistent with mass-loss rates amongst field stars of these types (Leitherer et al. 1995).

- Five of the 24 WRs known in Wd 1 were detected. Of these, WR L has a partially optically-thick wind, with a mass-loss rate consistent with stars of identical spectral type in the Galactic Centre cluster and the general field population. The remaining three (WR A, B and $\mathrm{V}$ ) are identified as having composite spectra from a CWB. The optical and X-ray properties of WR A and WR B have previously indicated these to be binaries, while this is the first hint of binarity for WR V.

In combination with X-ray, optical and near-IR datasets, these radio observations of $\mathrm{Wd} 1$ provide striking evidence for the prevalence of binarity among the massive stars in $\mathrm{Wd} \mathrm{1.} \mathrm{This}$ lends strong support to the previous estimates of high binary fractions, in excess of $>40 \%$ (C08, Ritchie et al. 2009a).

The rich stellar population of $\mathrm{Wd} 1$ permits us to investigate the evolution of mass-loss rates as stars evolve from the MS. With the reduction of O-star mass-loss rates due to wind clumping (e.g. Repolust et al. 2004), it has been recognised that the majority of mass loss must occur in the post-MS transitional phase. While the exact evolutionary sequence through the transitional "zoo" is uncertain for stars of $M_{\text {initial }} \sim 40 M_{\odot}$, it is interesting that the radio mass-loss rates directly determined for the YHG W4, the LBV W243 and the WNLh star WR L, which form a direct evolutionary sequence in some schemes, are all closely equal to one another. 
However, with the exception of W9, such mass-loss rates $\left(\leq 10^{-5} M_{\odot} \mathrm{yr}^{-1}\right)$ are likely insufficient to remove the H-rich mantle unless stars remain in the transitional phase for significantly longer than expected. This in turn suggests that an additional mechanism is required to shed the requisite mass, with short-lived episodes of greatly enhanced mass loss an obvious candidate. Indeed mass-loss rates $>10^{-4} M_{\odot} \mathrm{yr}^{-1}$ have already been inferred for RSGs (e.g. VY CMa; Smith et al. 2001) and directly observed for the YHG $\rho$ Cas (Lobel et al. 2003). The nebulae around the RSGs W20, 26 and 236 already indicate that significant mass loss has occurred for some stars within $\mathrm{Wd} 1$, while the mass-loss rate inferred for W9, over a magnitude greater than any other transitional star, is of obvious interest. Indeed, is W9 undergoing an "eruptive" event currently?

We note that both the optical spectrum and spectral energy distribution of W9 indicate that it is not a cool hypergiant, suggesting that extreme mass-loss events are likely not confined to a single evolutionary state, such as RSGs, and instead occur in both hot and cool transitional phases. Indeed long term spectroscopic observations indicate significant instability in the earlyB hypergiant/WNVL, RSG and YHG populations (Clark et al. 2010). Hence one may not a priori assume a single physical mechanism leads to enhanced mass loss in post-MS stars. When combined with detailed modeling of the stellar spectra, further observations to derive the nebular properties of cluster members, such as expansion velocity and chemical abundances, would be very valuable in order to constrain the phases in which enhanced mass loss occurs; thus Wd 1 will be invaluable for investigating the physics that mediates the passage of MS O-type stars to the Wolf-Rayet phase.

Acknowledgements. We thank Paul Crowther, Ben Davies, Simon Goodwin, Rene Oudmaijer, Julian Pittard, Ben Ritchie and Rens Waters for many stimulating discussions related to this work and for providing numerous comments on early versions of the manuscript. A special thanks to Rob Reid for advice on using the SMERF patch to DIFMAP and to Bob Sault for his extensive advise on the use of MIRIAD. The Australia Telescope Compact Array is part of the Australia Telescope which is funded by the Commonwealth of Australia for operation as a National Facility managed by CSIRO. SMD would like to thank the Anton Pannekoek Institute and Open University for their hospitality during a number of visits. JSC is supported by a UK Research Council (RCUK) Fellowship. This research is partially supported by the Spanish Ministerio de Ciencia e Innovación (MICINN) under grants AYA2008-06166-C03-03 and ConsoliderGTC CSD2006-70.

\section{Appendix A: Modelling radio emission from a circumstellar envelope}

For a spherically symmetric isothermal plasma envelope of temperature $T$ and outer radius $R_{\mathrm{o}}$ at distance $D$ from the Sun, the total flux at frequency $v$ is given by

$F_{v}=\frac{2 \pi}{D^{2}}\left(\frac{2 k T v^{2}}{c^{2}}\right) \int_{0}^{R_{\mathrm{o}}}\left(1-\mathrm{e}^{-\tau_{v}(p)}\right) p \mathrm{~d} p$,

where $\tau_{v}(p)$ is the optical depth along a line-of-sight at distance $p$ from the line-of-sight to the centre of the envelope ${ }^{3}$ (Fig. A.1) For a Maxwellian plasma in LTE the opacity is given by

$\tau_{v}=3.6 \times 10^{-2} T_{\mathrm{e}}^{-3 / 2} v^{-2} g_{v} \gamma Z^{2} \int_{0}^{\infty} n_{\mathrm{e}}^{2} \mathrm{~d} z$,

for electron temperature $T_{\mathrm{e}}, g_{v}$ the gaunt free-free factor (see e.g. Leitherer \& Robert 1991), $Z$ the rms charge of the plasma, $\gamma$ the ratio of electron to ions, and $n_{\mathrm{e}}$ is the electron density along a line-of-sight segment length $\mathrm{d} z$ (Rybicki \& Lightman 1986).

\footnotetext{
${ }^{3} p$ is often referred to as the "impact parameter".
}

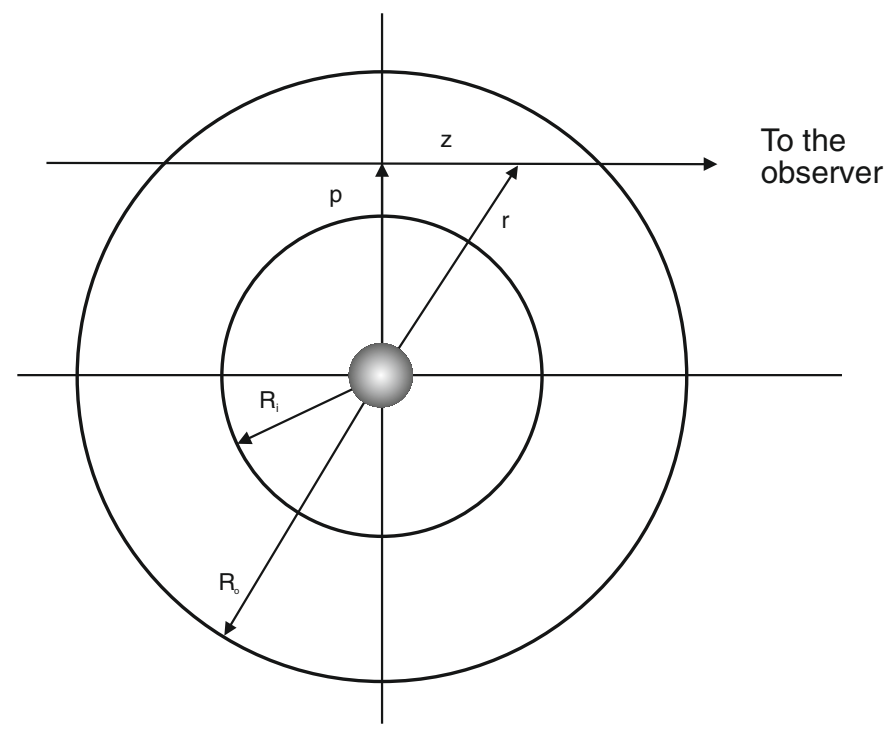

Fig. A.1. Geometry of a model circumstellar envelope with outer radius $R_{\mathrm{o}}$ and inner radius $R_{\mathrm{i}}$. For $R_{\mathrm{i}}<r<R_{\mathrm{o}}$ the plasma density $\propto r^{-2}$ and is zero for $r<R_{\mathrm{i}}$.

For a steady-state, constant velocity stellar wind of massloss rate $\dot{M}$ and terminal velocity $v_{\infty}$, the equation of continuity requires $n_{\mathrm{e}} v_{\infty} r^{2}=$ const., leading to

$$
n_{\mathrm{e}}=\frac{\dot{M}}{4 \pi v_{\infty} \mu m_{\mathrm{H}}}\left(\frac{1}{r^{2}}\right) \text {, }
$$

with $\mu$ the atomic weight of the plasma, and $m_{\mathrm{H}}$ the mass of hydrogen. The free-free opacity is then given by

$$
\tau_{v}(p)=A \int_{0}^{\infty} \frac{1}{\left(p^{2}+z^{2}\right)^{2}} \mathrm{~d} z=A I_{2}(p)
$$

where

$$
A=3.6 \times 10^{-2} T_{\mathrm{e}}^{-3 / 2} v^{-2} g_{v} \gamma\left(\frac{\dot{M} Z}{4 \pi v_{\infty} \mu m_{\mathrm{H}}}\right)^{2} .
$$

Analytic solutions for the integral $I_{2}(p)$ in Eq. (A.2) and the envelope geometry shown in Fig. A.1 can be derived, namely:

$$
\begin{aligned}
I_{2}(p)= & \frac{1}{2 p^{2}}\left[\frac{\left(R_{\mathrm{o}}^{2}-p^{2}\right)^{\frac{1}{2}}}{R_{\mathrm{o}}^{2}}-\frac{\left(R_{\mathrm{i}}^{2}-p^{2}\right)^{\frac{1}{2}}}{R_{\mathrm{i}}^{2}}\right] \\
& +\frac{1}{2 p^{3}}\left[\tan ^{-1}\left(\frac{\left(R_{\mathrm{o}}^{2}-p^{2}\right)^{\frac{1}{2}}}{p}\right)-\tan ^{-1}\left(\frac{\left(R_{\mathrm{i}}^{2}-p^{2}\right)^{\frac{1}{2}}}{p}\right)\right]
\end{aligned}
$$

if $0<p<R_{\mathrm{i}}$ and

$I_{2}(p)=\frac{1}{2 p^{3}}\left[\frac{p\left(R_{\mathrm{o}}^{2}-p^{2}\right)^{\frac{1}{2}}}{R_{\mathrm{o}}^{2}}+\tan ^{-1}\left(\frac{\left(R_{\mathrm{o}}^{2}-p^{2}\right)^{\frac{1}{2}}}{p}\right)\right]$

if $R_{\mathrm{i}}<p<R_{\mathrm{O}}$.

Determining the flux from an extended nebula at any given frequency requires using Eq. (A.2) and the two conditional cases for $\left.I_{2}\right) p$ ) given by Eqs. (A.3) and (A.4) to determine the opacity along a particular line-of-sight, before solving Eq. (A.1). To model the flux from a "standard" stellar wind, $R_{\mathrm{i}}$ is set to the radius of the underlying star $R_{*}$ (for the models considered here $R_{*} \ll R_{\mathrm{o}}$, so it is assumed that $\left.R_{\mathrm{i}} \equiv 0\right)$ and only the conditional case for $I_{2}(p)$ given in Eq. (A.4) needs to be considered. 


\section{References}

Abbott, D. C., Bieging, J. H., \& Churchwell, E. 1981, ApJ, 250, 645 Benjamin, R. A., Churchwell, E., Babler, B. L., et al. 2003, PASP, 115, 953 Berghoefer, T. W., Schmitt, J. H. M. M., Danner, R., \& Cassinelli, J. P. 1997, A\&A, 322, 167

Blöcker, T., Balega, Y., Hofmann, K.-H., et al. 1999, A\&A, 348, 805

Blöcker, T., Balega, Y., Hofmann, K.-H., \& Weigelt, G. 2001, A\&A, 369, 142

Blundell, K. M., Mioduszewski, A. J., Muxlow, T. W. B., Podsiadlowski, P., \& Rupen, M. P. 2001, ApJ, 562, L79

Bonanos, A. Z. 2007, AJ, 133, 2696

Borgman, J., Koornneef, J., \& Slingerland, J. 1970, A\&A, 4, 248

Brandner, W., Clark, J. S., Stolte, A., et al. 2008, A\&A, 478, 137

Cappa, C., Goss, W. M., \& van der Hucht, K. A. 2004, AJ, 127, 2885

Castro-Carrizo, A., Quintana-Lacaci, G., Bujarrabal, V., Neri, R., \& Alcolea, J. 2007, A\&A, 465, 457

Chapman, J. M., Leitherer, C., Koribalski, B., Bouter, R., \& Storey, M. 1999, ApJ, 518, 890

Clark, J. S., \& Negueruela, I. 2002, A\&A, 396, L25

Clark, J. S., \& Porter, J. M. 2004, A\&A, 427, 839

Clark, J. S., Fender, R. P., Waters, L. B. F. M., et al. 1998, MNRAS, 299, L43

Clark, J. S., Egan, M. P., Crowther, P. A., et al. 2003, A\&A, 412, 185

Clark, J. S., Negueruela, I., Crowther, P. A., \& Goodwin, S. P. 2005, A\&A, 434, 949

Clark, J. S., Barnes, A. D., \& Charles, P. A. 2007, MNRAS, 380, 263

Clark, J. S., Muno, M. P., Negueruela, I., et al. 2008, A\&A, 477, 147

Clark, J. S., Crowther, P. A., Larionov, V. M., et al. 2009, A\&A, 507, 1555

Clark, J. S., Ritchie, B. W., \& Negueruela, I. 2010, A\&A, accepted

Corcoran, M. F. 2008, in Rev. Mex. Astron. Astrofis. Conf. Ser., 33, 123

Crowther, P. A., Hadfield, L. J., Clark, J. S., Negueruela, I., \& Vacca, W. D. 2006, MNRAS, 372, 1407

de Jager, C. 1998, A\&A Rev., 8, 145

de Martino, D., Vittone, A. A., Rossi, C., \& Giovannelli, F. 1992, A\&A, 254, 266

de Wit, W. J., Oudmaijer, R. D., Fujiyoshi, T., et al. 2008, ApJ, 685, L75

Dougherty, S. M., \& Williams, P. M. 2000, MNRAS, 319, 1005

Dougherty, S. M., Beasley, A. J., Claussen, M. J., Zauderer, B. A., \& Bolingbroke, N. J. 2005, ApJ, 623, 447

Duncan, R. A., \& White, S. M. 2002, MNRAS, 330, 63

Fullerton, A. W., Massa, D. L., \& Prinja, R. K. 2006, ApJ, 637, 1025

Gehrz, R. D., Hayward, T. L., Houck, J. R., et al. 1995, ApJ, 439, 417

Groh, J. H., Damineli, A., Teodoro, M., \& Barbosa, C. L. 2006, A\&A, 457, 591

Groh, J. H., Hillier, D. J., Damineli, A., et al. 2009, ApJ, 698, 1698

Higgs, L. A., Feldman, P. A., \& Smolinski, J. 1978, ApJ, 220, L109

Hoare, M. G., Kurtz, S. E., Lizano, S., Keto, E., \& Hofner, P. 2007, in Protostars and Planets V, ed. B. Reipurth, D. Jewitt, \& K. Keil, 181

Ignace, R., \& Churchwell, E. 2004, ApJ, 610, 351

Israelian, G., Lobel, A., \& Schmidt, M. R. 1999, ApJ, 523, L145

Jura, M., \& Kleinmann, S. G. 1990, ApJS, 73, 769

Kothes, R., \& Dougherty, S. M. 2007, A\&A, 468, 993

Lang, C. C. 2003, IAU Symp., ed. K. van der Hucht, A. Herrero, \& C. Esteban, 497

Leitherer, C., \& Robert, C. 1991, ApJ, 377, 629

Leitherer, C., Chapman, J. M., \& Koribalski, B. 1995, ApJ, 450, 289

Leitherer, C., Chapman, J. M., \& Koribalski, B. 1997, ApJ, 481, 898

Lobel, A., Israelian, G., de Jager, C., et al. 1998, A\&A, 330, 659

Lobel, A., Dupree, A. K., Stefanik, R. P., et al. 2003, ApJ, 583, 923

Martins, F., Schaerer, D., Hillier, D. J., et al. 2005, A\&A, 441, 735

Martins, F., Genzel, R., Hillier, D. J., et al. 2007, A\&A, 468, 233

Mengel, S., \& Tacconi-Garman, L. E. 2007, A\&A, 466, 151

Moffat, A. F. J., Corcoran, M. F., Stevens, I. R., et al. 2002, ApJ, 573, 191
Mokiem, M. R., de Koter, A., Vink, J. S., et al. 2007, A\&A, 473, 603

Monnier, J. D., Tuthill, P. G., \& Danchi, W. C. 1999, ApJ, 525, L97

Morris, M., \& Jura, M. 1983, ApJ, 267, 179

Muno, M. P., Clark, J. S., Crowther, P. A., et al. 2006a, ApJ, 636, L41

Muno, M. P., Law, C., Clark, J. S., et al. 2006b, ApJ, 650, 203

Najarro, F., Figer, D. F., Hillier, D. J., Geballe, T. R., \& Kudritzki, R. P. 2009, ApJ, 691, 1816

Negueruela, I., \& Clark, J. S. 2005, A\&A, 436, 541

Negueruela, I., Clark, J. S., \& Ritchie, B. W. 2010, A\&A, submitted

Oudmaijer, R. D., Davies, B., de Wit, W., \& Patel, M. 2009, ed.

D. G. Luttermoser, B. J. Smith, \& R. E. Stencel, ASP Conf. Ser., 412, 17

Oudmaijer, R. D., Groenewegen, M. A. T., Matthews, H. E., Blommaert,

J. A. D. L., \& Sahu, K. C. 1996, MNRAS, 280, 1062

Patel, M., Oudmaijer, R. D., Vink, J. S., et al. 2008, MNRAS, 385, 967

Pittard, J. M. 2009, MNRAS, 396, 1743

Pittard, J. M., Dougherty, S. M., Coker, R. F., O'Connor, E., \& Bolingbroke,

N. J. 2006, A\&A, 446, 1001

Pittard, J. M., \& Parkin, E. R. 2009, MNRAS, accepted [arXiv: 0909. 4383]

Povich, M. S., Stone, J. M., Churchwell, E., et al. 2007, ApJ, 660, 346

Reid, R. I. 2006, MNRAS, 367, 1766

Repolust, T., Puls, J., \& Herrero, A. 2004, A\&A, 415, 349

Ritchie, B. W., Clark, J. S., Negueruela, I., \& Crowther, P. A. 2009a, A\&A, 507, 1585

Ritchie, B. W., Clark, J. S., Negueruela, I., \& Najarro, F. 2009b, A\&A, 507, 1597

Ritchie, B. W., Clark, J. S., \& Negueruela, I. 2010, A\&A, submitted

Rybicki, G. B., \& Lightman, A. P. 1986, Radiative Processes in Astrophysics, ed. G. B. Rybicki, \& A. P. Lightman

Sault, R. J., Teuben, P. J., \& Wright, M. C. H. 1995, in Astronomical Data Analysis Software and Systems IV, ASP Conf. Ser., 77, 433

Schuster, M. T., Humphreys, R. M., \& Marengo, M. 2006, AJ, 131, 603

Serabyn, E., Lacy, J. H., \& Achtermann, J. M. 1991, ApJ, 378, 557

Setia Gunawan, D. Y. A., Chapman, J. M., Stevens, I. R., Rauw, G., \& Leitherer,

C. 2003, ed. K. van der Hucht, A. Herrero, \& C. Esteban, IAU Symp., 230

Shepherd, M. C. 1997, in Astronomical Data Analysis Software and Systems VI, ASP Conf. Ser., 125, 77

Skinner, S. L., Simmons, A. E., Zhekov, S. A., et al. 2006, ApJ, 639, L35

Smith, N., \& Owocki, S. P. 2006, ApJ, 645, L45

Smith, N., Humphreys, R. M., Davidson, K., et al. 2001, AJ, 121, 1111

Stahl, O., Wolf, B., Aab, O., \& Smolinski, J. 1991, A\&A, 252, 693

Stevens, I. R., Blondin, J. M., \& Pollock, A. M. T. 1992, ApJ, 386, 265

Stickland, D. J., \& Harmer, D. L. 1978, A\&A, 70, L53

Sylvester, R. J., Skinner, C. J., \& Barlow, M. J. 1998, MNRAS, 301, 1083

Tafoya, D., Gómez, Y., \& Rodríguez, L. F. 2004, ApJ, 610, 827

Taylor, A. R., Pottasch, S. R., \& Zhang, C. Y. 1987, A\&A, 171, 178

Tuthill, P. G., Monnier, J. D., \& Danchi, W. C. 1999, Nature, 398, 487

van der Hucht, K. A., Schrijver, H., Stenholm, B., et al. 1997, New Astron., 2, 245

van Loo, S., Runacres, M. C., \& Blomme, R. 2006, A\&A, 452, 1011

Voors, R. H. M., Waters, L. B. F. M., de Koter, A., et al. 2000, A\&A, 356, 501

Watson, C., Povich, M. S., Churchwell, E. B., et al. 2008, ApJ, 681, 1341

Westerlund, B. 1961, PASP, 73, 51

Westerlund, B. E. 1987, A\&AS, 70, 311

Westmoquette, M. S., Exter, K. M., Smith, L. J., \& Gallagher, J. S. 2007, MNRAS, 381, 894

Williams, P. M. 1999, in Wolf-Rayet Phenomena in Massive Stars and Starburst

Galaxies, ed. K. A. van der Hucht, G. Koenigsberger, \& P. R. J. Eenens, IAU Symp., 193, 267

Williams, P. M., Dougherty, S. M., Davis, R. J., et al. 1997, MNRAS, 289, 10

Williams, P. M., Marchenko, S. V., Marston, A. P., et al. 2009, MNRAS, 395, 1749

Yusef-Zadeh, F., \& Morris, M. 1991, ApJ, 371, L59 